\title{
Hippocampal and striatal responses during motor learning are modulated by prefrontal cortex stimulation
}

\author{
Mareike A. Gann ${ }^{\mathrm{a}, \mathrm{b}}$, Bradley R. King ${ }^{\mathrm{a}, \mathrm{b}}$, Nina Dolfen ${ }^{\mathrm{a}, \mathrm{b}}$, Menno P. Veldman ${ }^{\mathrm{a}, \mathrm{b}}$, \\ Kimberly L. Chan ${ }^{c}$, Nicolaas A.J. Puts ${ }^{\mathrm{d}, e, f}$, Richard A.E. Edden ${ }^{\mathrm{d}, \mathrm{e}}$, Marco Davare ${ }^{\mathrm{g}}$, \\ Stephan P. Swinnen ${ }^{\mathrm{a}, \mathrm{b}}$, Dante Mantini ${ }^{\mathrm{a}}$,h , Edwin M. Robertson ${ }^{\mathrm{i}}$, Geneviève Albouy ${ }^{\mathrm{a}, \mathrm{b}, *}$ \\ ${ }^{a}$ Department of Movement Sciences, Movement Control and Neuroplasticity Research Group, KU Leuven, 3001 Leuven, Belgium \\ ${ }^{\mathrm{b}}$ LBI - KU Leuven Brain Institute, KU Leuven, 3001 Leuven, Belgium \\ c Advanced Imaging Research Center, University of Texas Southwestern, Dallas, TX 75390, USA \\ ${ }^{\mathrm{d}}$ The Russell H. Morgan Department of Radiology and Radiological Science, The Johns Hopkins University School of Medicine, Baltimore, MD 21205, USA \\ ${ }^{\mathrm{e}}$ F. M. Kirby Research Center for Functional Brain Imaging, Kennedy Krieger Institute, Baltimore, MD 21205, USA \\ ${ }_{\mathrm{f}}^{\mathrm{f}}$ Department of Forensic and Neurodevelopmental Sciences and the Institute of Psychiatry, Psychology, and Neuroscience; King's College London, SE5 8AF London, \\ United Kingdom \\ ${ }^{\mathrm{g}}$ Department of Clinical Sciences, College of Health and Life Sciences, Brunel University London, UB8 3PN Uxbridge, United Kingdom \\ ${ }^{\mathrm{h}}$ Brain Imaging and Neural Dynamics Research Group, IRCCS San Camillo Hospital, 30126 Venice, Italy \\ ${ }^{\mathrm{i}}$ Institute of Neuroscience and Psychology, University of Glasgow, G12 8QB Glasgow, United Kingdom
}

\section{A R T I C L E I N F O}

\section{Keywords:}

Theta-burst stimulation

Motor learning

Prefrontal cortex

Hippocampus

Striatum

GABA

\begin{abstract}
A B S T R A C T
While it is widely accepted that motor sequence learning (MSL) is supported by a prefrontal-mediated interaction between hippocampal and striatal networks, it remains unknown whether the functional responses of these networks can be modulated in humans with targeted experimental interventions. The present proof-of-concept study employed a multimodal neuroimaging approach, including functional magnetic resonance (MR) imaging and MR spectroscopy, to investigate whether individually-tailored theta-burst stimulation of the dorsolateral prefrontal cortex can modulate responses in the hippocampus and the basal ganglia during motor learning. Our results indicate that while stimulation did not modulate motor performance nor task-related brain activity, it influenced connectivity patterns within hippocampo-frontal and striatal networks. Stimulation also altered the relationship between the levels of gamma-aminobutyric acid (GABA) in the stimulated prefrontal cortex and learning-related changes in both activity and connectivity in fronto-striato-hippocampal networks. This study provides the first experimental evidence, to the best of our knowledge, that brain stimulation can alter motor learning-related functional responses in the striatum and hippocampus.
\end{abstract}

\section{Introduction}

The neural responses underlying motor sequence learning (MSL) have been thoroughly investigated and various models propose that this process is supported by cortico-cerebellar, -striatal and -hippocampal networks (Doyon et al., 2009; Penhune and Steele, 2012; Albouy et al., 2013a). Interestingly, these brain systems present different dynamical patterns of activity during the learning process (Albouy et al., 2013a). Whereas activity in hippocampo-fronto-parietal networks, which form loops with associative regions of the striatum and the cerebellum, decreases as a function of learning, activity in sensorimotor circuits, including the sensorimotor parts of the striatum, the cerebellum and motor cortical areas, increases with learning (Hikosaka et al., 2002;
Albouy et al., 2013a; Doyon et al., 2018). Importantly, functional connectivity between these networks reveals a competitive interaction pattern during initial learning (Albouy et al., 2013a, 2013b). Crucial to the present study, the interaction between hippocampal and striatal systems is orchestrated by the dorsolateral prefrontal cortex (DLPFC) (Albouy et al., 2012, 2013a,b; Freedberg et al., 2020).

As the hippocampal and striatal neural signatures described above are thought to support motor memory acquisition and also predict successful motor memory retention (Albouy et al., 2008, 2013b; Steele and Penhune, 2010), investigating whether these learning-related brain responses can be altered by experimental interventions is of the utmost importance. One experimental approach that has shown promise to modulate neural responses in the striatum and hippocampus is the application

\footnotetext{
* Corresponding author at: Movement Control and Neuroplasticity Research Group, Department of Movement Sciences, KU Leuven, Tervuursevest 101 - Box 1501 , 3001 Leuven, Belgium.

E-mail address: genevieve.albouy@kuleuven.be (G. Albouy).
} 
of transcranial magnetic stimulation (TMS) to cortical regions that are functionally connected to these deeper areas. It has been shown that the application of repetitive TMS to the DLPFC or the parietal cortex can alter hippocampal activity and connectivity patterns which, in turn, influences performance on declarative memory tasks (Bilek et al., 2013; Wang et al., 2014; Kim et al., 2018; Hermiller et al., 2020; Thakral et al., 2020). As the role of the hippocampus in motor memory has been overlooked in the past [for discussion, see (Albouy et al., 2013a)], research aiming at modulating the motor learning process via hippocampaltargeted brain stimulation is lacking. Furthermore, prefrontal TMS has also been shown to influence striatal activity and connectivity during reward processing (van Holstein et al., 2018) and probabilistic learning (Ott et al., 2011). However, no such evidence is available in the motor memory domain, which is surprising given the critical role the striatum plays in this process. Based on the aforementioned evidence that the DLPFC mediates the interaction between the striato- and hippocampocortical systems during initial MSL and that prefrontal stimulation can influence functional responses in these networks, the DLPFC is a promising cortical stimulation target in order to alter brain responses in motor learning-relevant networks.

The goal of the present proof-of-concept study was therefore to use an extensive and multimodal neuroimaging approach, including functional Magnetic Resonance Imaging (fMRI) and MR Spectroscopy (MRS), to test whether stimulation of the DLPFC can modulate motor-learningrelated functional responses in the basal ganglia and the hippocampus. Based on evidence that the neuromodulatory effects of TMS can be optimized by defining stimulation targets via data-driven approaches and tailoring the stimulation targeting procedures to each individual (Sack et al., 2009; Fox et al., 2012a,b; Beynel et al., 2019), the TMS target in the present study was identified using a functional-data-driven approach tailored to each individual. To do so, we analyzed fMRI data from a sample of individuals independent from the current sample and identified a cortical cluster functionally connected to both the striatum and hippocampus at rest. The spatial location of this cortical cluster was used to guide the individualized TMS targeting procedure used in the present study.

Repetitive TMS was applied with a theta burst stimulation (TBS) procedure to the individually-identified prefrontal cortical target before participants were trained on a sequential serial reaction time task (SRTT; (Nissen and Bullemer, 1987)) or a control random condition (random SRTT). Using a region of interest (ROI) approach, we examined the effect of intermittent versus continuous TBS (i.e., iTBS and cTBS, respectively; (Huang et al., 2005)) of the DLPFC on (i) task-related activity and connectivity patterns in fronto-striato-hippocampal networks measured with fMRI during post-stimulation task practice and (ii) DLPFC neurochemistry through the quantification of gamma-aminobutyric acid (GABA), the brain's primary inhibitory neurotransmitter, pre- and postintervention using MRS.

Based on previous behavioral research showing that disruptive DLPFC stimulation can effectively impair motor learning processes (Pascual-Leone et al., 1996; Robertson et al., 2001; Burke and Coats, 2016; Dayan et al., 2018), we expected inhibitory cTBS of the DLPFC to disrupt motor sequence learning as compared to facilitatory iTBS. At the brain level, as stimulation-induced effects of TBS on neural excitability have been shown to be similar in the prefrontal cortex as in the primary motor cortex (M1; (Chung et al., 2017)), we hypothesized that facilitatory iTBS and inhibitory cTBS of the DLPFC would respectively strengthen and disrupt activity and connectivity in hippocampoprefrontal networks during sequence learning as compared to random practice. Based on models suggesting that hippocampo-prefrontal networks exert control processes over sensorimotor-striato-cortical networks during MSL (Albouy et al., 2013a), we expected that facilitatory iTBS and inhibitory cTBS of the DLPFC would repress and facilitate, respectively, the development of striato-motor activity during sequence learning. With respect to GABA measures, which are thought to reflect activity of inhibitory GABAergic interneurons in the neocor-
Table 1

Participant characteristics.

\begin{tabular}{ll}
\hline $\mathrm{N}$ & $19(12$ females $)$ \\
Age (years) & $22.42( \pm 2.36)$ \\
Beck Anxiety Inventory & $1.68( \pm 2.43)$ \\
Beck Depression Inventory & $3.84( \pm 3.95)$ \\
Edinburgh Handedness & $85.26( \pm 14.57)$ \\
Pittsburgh Sleep Quality Index & $3.52( \pm 1.54)$ \\
St. Mary Quality of sleep & $4.04( \pm 0.8)$ \\
St. Mary Quantity of sleep & $8 \mathrm{~h}( \pm 1.01)$ \\
Stanford Sleepiness Scale & $2.11( \pm 0.76)$ \\
\hline
\end{tabular}

Group means \pm SD for participant characteristics, standardized questionnaires as well as the vigilance assessments administered at time of testing for included participants. Data of Stanford Sleepiness Scale and St. Mary Questionnaire were averaged across conditions (see Supplemental Table S1 for within condition data; see Supplemental Tables S2 and S3 for stimulation and task effects on vigilance and sleep).

tex (Tremblay et al., 2016), previous MRS studies have shown that M1 GABA levels can be altered by both M1 brain stimulation (Stagg et al., 2009b,a, 2011a; Marjańska et al., 2013; Bachtiar et al., 2015, 2018) and motor learning (Floyer-Lea et al., 2006; Sampaio-Baptista et al., 2015; Kolasinski et al., 2018). However, less is known about effects of motor learning and brain stimulation on prefrontal GABA (HoneBlanchet et al., 2016; Iwabuchi et al., 2017). Similar to previous M1 studies, we hypothesized that facilitatory iTBS and inhibitory cTBS of the DLPFC would result in a decrease and increase in DLPFC GABA, respectively; and these effects would be more pronounced for sequence learning as compared to the control task. As GABA levels are typically inversely related to BOLD signal (Duncan et al., 2014), we expected that the intervention-related modulation of activity and connectivity described above would be negatively correlated to the hypothesized changes in DLPFC GABA levels.

\section{Methods}

\subsection{Ethics statement}

This experiment was approved by the local Ethics Committee (UZ / KU Leuven). All participants gave their written informed consent before taking part in the study and were compensated for their participation. Procedures were executed in conformity with the approved guidelines.

\subsection{Participants}

Twenty-one young (range: 19-26 years) right-handed (Oldfield, 1971) participants took part in this study. All participants had normal or corrected-to-normal vision, were nonsmokers, free of psychoactive (e.g., anti-depressant or -anxiety) medications, reported no known psychological, psychiatric or neurological disorders [including anxiety (Beck et al., 1988) and depression (Beck et al., 1961)], and had no contra-indications for MRI or TMS. Furthermore, none of the participants were considered musicians or professional typists. The quality and quantity of sleep during the month preceding the experiment was normal as assessed by the Pittsburgh Sleep Quality Index (Buysse et al., 1989). Two participants were excluded because of incidental findings on the acquired imaging data. Nineteen participants were eventually included in the final analyses (see participants' characteristics in Table 1, and Supplemental Table S1 for within condition sleep and vigilance scores). Due to technical problems, one experimental session (out of four) is missing for one participant. Behavioral, MRS and MRI data of two experimental sessions were excluded for another participant as he/she failed to appropriately perform the motor task (i.e., $>3$ SD below the mean for accuracy). One session 
of another participant was excluded from the fMRI analyses due to excessive head motion (i.e., $>2$ voxels). Motor Evoked Potential (MEP) data are missing for one participant. Consequently, behavioral, MEP, MRS and MRI analyses included 16 to 18 participants depending on the contrasts and conditions tested. Note that due to the multimodal nature of the present study, the choice of a specific outcome (among motor behavior, task-related activity, task- and resting-state-related connectivity, GABA levels) to perform sample size computation could be considered arbitrary. Consequently, our sample size estimation was based on previous studies that also sought to alter functional responses in deep areas via non-invasive brain stimulation applied to cortical targets. Previous research included on average 20 participants per group (van der Werf et al., 2010; Ott et al., 2011; Bilek et al., 2013; Esslinger et al., 2014; Wang et al., 2014; Hanlon et al., 2016; van Holstein et al., 2018; Alkhasli et al., 2019; Freedberg et al., 2019), thus, this was our targeted sample size.

\subsection{General experimental procedure}

Participants were invited to complete five experimental sessions (one baseline and four TBS sessions) at the University Hospital of KU Leuven. All sessions occurred between 9am and 6pm. Moreover, all five sessions completed by each participant took place at approximately the same time of the day $( \pm 2 \mathrm{~h}$ ) to minimize the influence of circadian phase variation on behavior (Smarr et al., 2014), brain function (Muto et al., 2016) and brain excitability (de Beukelaar et al., 2016). TBS sessions were separated by at least 6 days (mean time between stimulation sessions: $7.9 \pm 2.9$ days) to avoid carry-over effects. Participants were instructed to have a good night of sleep before each experimental session and to avoid alcohol consumption the day before and the day of the experimental session. Sleep quality and quantity of the nights before each experimental session were assessed with the St. Mary's Hospital Sleep Questionnaire ((Ellis et al., 1981), see Table 1). Vigilance at the time of testing was assessed subjectively at the beginning of each session using the Stanford Sleepiness Scale (SSS; (Maclean et al., 1992)). There were no differences in sleep quantity and quality of the night preceding each condition (all Fs $<3.647$, all $p s>.073$, see Supplemental Table S2) and differences in subjective vigilance (see Supplemental Table S2) did not influence performance speed in any condition (all $p s>.353$, see Supplemental Table S3 and Supplemental Results).

During the baseline MR session, a high-resolution T1-weighted image (to be subsequently used for neuronavigated TMS), RS functional data (to identify individual TBS targets, see below) as well as diffusionweighted images (not reported in this manuscript) were acquired. Participants were also trained - for habituation purposes - on a random version of the serial reaction time task (see below). The session ended with a series of measures using the TMS equipment (determining the hot spot, resting and active motor thresholds, and corticospinal excitability through MEPs, see "TMS administration" section). The next four experimental sessions were organized according to a stimulation (2 levels: intermittent TBS [iTBS] vs. continuous TBS [cTBS]) by task (2 levels: sequence [SEQ] vs. random [RND]) within-subject design (Fig. 1; see below for details on the stimulation and task conditions). In each session, participants first underwent pre-TBS RS and MRS scans of the DLPFC and the hippocampus (see below for acquisition details) that were followed by T1-neuronavigated TBS applied to an individually-defined DLPFC target (see individual target identification below) outside the scanner. MEPs were measured pre- and post-stimulation as described below. Immediately following the end of the stimulation session, participants were placed in the MRI scanner where they were trained on the motor task while BOLD images were acquired (mean delay between start TBS and start task: 15.71 min, range 12-22; mean duration of the task training: $11.5 \mathrm{~min}$, range 9.33-13.43). After task completion, post-TBS/task RS and MRS data of the DLPFC and hippocampus were acquired (intervals between TBS and post-TBS/task DLPFC and hippocampus MRS were $40.2 \mathrm{~min}$, range: $36-46$ and $51.85 \mathrm{~min}$, range: $48-57$, respectively; inter- vals between end of the task and post-TBS/task DLPFC and hippocampus MRS were $12.65 \mathrm{~min}$, range: $12-15$ and $24.29 \mathrm{~min}$, range: $24-26$, respectively). The order of the four experimental conditions [CTBS/SEQ (cSEQ), cTBS/RND (cRND), iTBS/SEQ (iSEQ), iTBS/RND (iRND)] was counterbalanced across the 21 participants. It is important to note however that due to participant / data exclusion during data analyses (see participant section), the distribution of the different conditions per visit was not balanced in the analyzed sample (see Supplemental Table S4 for the distribution of the different conditions per visit). This was taken into account in additional control behavioral, MRS and MRI data analyses (see below).

\subsection{Serial reaction time task}

An explicit bimanual version of the serial reaction time task (SRTT; (Nissen and Bullemer, 1987)) previously used in our group (King et al., 2019) that was coded and implemented with the Psychophysics Toolbox in Matlab (Brainard, 1997) was used in this study. Participants were lying in the scanner with a specialized MR-compatible keyboard placed on their lap. During the task, eight squares were presented on the screen via a mirror above the participant's head. Each square corresponded spatially to one of the eight keys on the keyboard and to one of eight fingers (excluding thumbs). The color of the outline of the squares alternated between red and green, indicating rest and practice blocks, respectively. After each rest block (15 s), the outlines of all squares changed from red to green, indicating that participants should prepare to perform the task. Subsequently, one of the eight squares was colored (i.e., filled) green, and participants were instructed to press the corresponding key with the corresponding finger as fast and as accurately as possible. As soon as a key was pressed, regardless of whether the response was correct or not, the next square in a sequence changed to green (response to stimulus interval $=0 \mathrm{~ms}$ ). Each block of practice included 48 key presses and each training session included 16 blocks. Depending on the specific experimental condition, the order in which the squares were filled green (and thus the order of finger movements) followed either a pseudorandom (RND) or a fixed, repeating sequential pattern (SEQ). During the sequence conditions, participants performed one of two eight-element sequences (whereby each of the eight fingers was pressed once in a sequence) that was repeated six times per block. The sequences were 4-73-8-6-2-5-1 and 7-2-8-4-1-6-3-5 with 1 representing the left little finger and 8 representing the right little finger, respectively. Note that due to experimental error, one participant was trained on sequences 4-7-3-8-62-5-1 and 2-6-1-5-8-3-7-4 and one participant was trained on 7-2-8-4-16-3-5 and 2-6-1-5-8-3-7-4. In the pseudorandom condition, there was no repeating sequence, but each key was pressed once every eight elements (i.e., no repeating elements); thus, each finger was also used six times per block. Participants were explicitly informed when the stimuli would follow a random pattern or a repeating sequential pattern but, in the latter case, they were not given any additional information such as what the pattern was or how many elements the sequence was composed of.

Mean response time for correct responses (RT, reflecting performance speed) and percentage of correct responses (percentage correct, reflecting movement accuracy) were computed for each block. Data were analyzed using repeated measures analyses of variance (ANOVAs; $\alpha=.05$ ) with stimulation (cTBS and iTBS), task (SEQ and RND) as well as block (1-16) as within-subject factors. Greenhouse-Geisser corrections were applied in case of violation of the sphericity assumption. Additional control analyses were performed using linear mixed models in order to take into account any potential visit effect (see Supplemental Results).

\subsection{TMS administration}

Based on evidence that TMS effects can be optimized by (i) the definition of stimulation targets using data-driven approaches and by (ii) individualized targeting procedures (Sack et al., 2009; Fox et al., 2012a,b; 


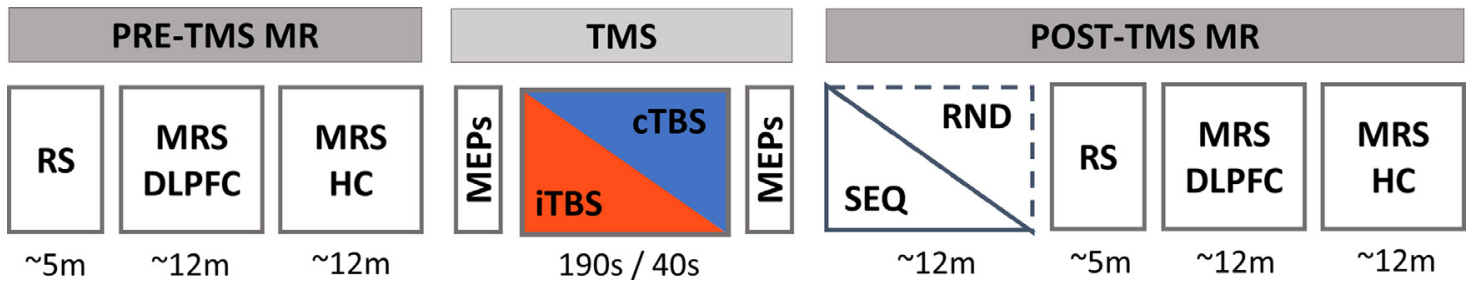

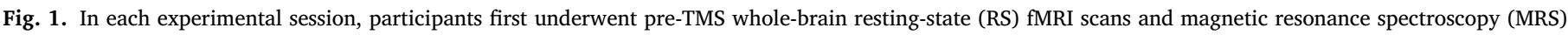

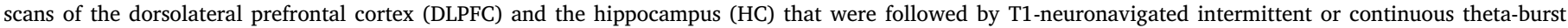

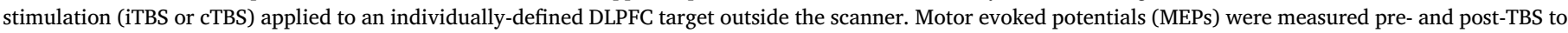

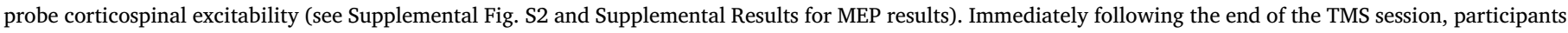

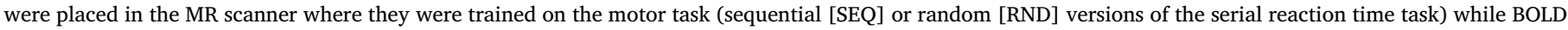

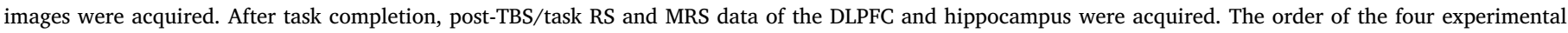

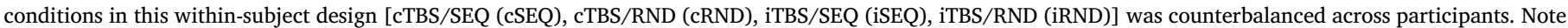
that the data related to the pre- and post-TBS RS scans are not reported in the present manuscript. TMS: transcranial magnetic stimulation.

Beynel et al., 2019), the TMS target in the present study was identified using a 2 step-approach. First, we analyzed fMRI data from a sample of young healthy individuals independent from the sample of the current study and identified a cortical cluster functionally connected to both the striatum and hippocampus at rest (see details in "Group target identification on an independent sample of participants" section below). In a second step, the spatial location of this cortical cluster was used to guide the individualized TMS targeting procedure used in the current study (see "Individual target identification using baseline RS data").

\subsection{Group target identification on an independent RS fMRI dataset}

RS fMRI data already available in the lab (King et al., 2018) from a sample of 29 young healthy individuals (independent from the current sample) were analyzed in order to identify a cortical target to be used in the current experiment. Information on participants and RS fMRI data acquisition and analyses can be found in the Supplemental Material. Briefly, the goal of the connectivity analyses performed on this dataset was to identify cortical regions reachable using TBS that were functionally and commonly connected to both the striatum and the hippocampus. To do so, we performed whole-brain FC analyses using the hippocampus and caudate nucleus (bilaterally, as defined anatomically according to the AAL brain atlas; (Tzourio-Mazoyer et al., 2002)) as seeds. Note that the striatal seed was restricted to the caudate nucleus, as this region exhibits functional and anatomical connectivity with the DLPFC (Lehéricy et al., 2004; Albouy et al., 2012), the TBS target region. For each individual and for each seed, the time-series across all voxels within the seed were averaged and Pearson correlation coefficients with all the voxels of the brain were computed. To ensure normality, each correlation coefficient was Fishers r-to-z transformed using the formula $\mathrm{z}=\operatorname{arctanh}(\mathrm{r})$. Statistical analyses were performed on the $\mathrm{z}$-values and were based on comparisons of the correlation coefficients to a value of 0 . Statistical probabilities were considered significant if surviving the false discovery rate (FDR) method for multiple comparisons $\left(p_{\mathrm{FDR}}<.05\right)$. A conjunction analysis testing the "Conjunction Null Hypothesis" was performed between the hippocampal and striatal FDR-corrected connectivity Z-maps (Fig. 2A and Supplemental Table S5, hippocampus: Z $\geq 2.03$, $p_{\text {FDR }}<.05$; caudate: $\left.\mathrm{Z} \geq 1.996, p_{\mathrm{FDR}}<.05\right)$ using the easythresh_conj function (Nichols, 2007) rendering the conjunction map onto an average brain template provided by FSL (www.fmrib.ox.ac.uk/fsl, avg152T1) and thresholded at the highest $Z$ score of both RSFC maps $(Z=2.03)$. The resulting statistical map showed that a network including ventral medial prefrontal, dorsolateral prefrontal, parietal and subcortical regions was significantly and commonly connected to both seed regions. Based on evidence reviewed above that (i) the DLPFC plays a pivotal role in the interaction between hippocampal and striatal systems during MSL (Albouy et al., 2013a) and that (ii) repetitive TMS of the DLPFC can influence brain responses in these deep regions (e.g. (Ott et al., 2011;
Bilek et al., 2013)), we constrained our TBS target search on the conjunction map to a mask including the middle and superior frontal segments of the AAL atlas (Tzourio-Mazoyer et al., 2002). The resulting masked statistical map is shown in Fig. 2B and the list of identified frontal peaks is presented in Supplemental Table S6. The stimulation target - to be used in the present experiment to guide the individualized targeting pipeline - was defined as the peak maxima in the masked conjunction map and was located in the left DLPFC (-30 $2248 \mathrm{~mm}$, encircled in black in Fig. 2B).

\subsection{Individual target identification using baseline RS data}

Individual TBS targets were identified using each participant's RS data collected during the baseline session. RS fMRI data were acquired on a Philips Achieva 3.0T MRI system equipped with a 32-channel head coil using an ascending gradient EPI pulse sequence for T2*-weighted images $\left(\mathrm{TR}=1000 \mathrm{~ms}\right.$; $\mathrm{TE}=33 \mathrm{~ms}$; multiband factor 3; flip angle $=80^{\circ}$; 42 transverse slices; interslice gap $=0.5 \mathrm{~mm}$; voxel size $=2.15 \times 2.14 \times 3$ $\mathrm{mm}^{3}$; field of view $=240 \times 240 \times 146.5 \mathrm{~mm}^{3}$; matrix $=112 \times 110 ; 300$ dynamic scans). Note that due to multiband capacity failure, the baseline RS data of one participant had different parameters: TR $=2500 \mathrm{~ms}$; $\mathrm{TE}=30 \mathrm{~ms}$; flip angle $=90^{\circ} ; 45$ transverse slices; slice thickness $=3$ $\mathrm{mm}$; interslice gap $=0.25 \mathrm{~mm}$; voxel size $2.5 \times 2.56 \times 3 \mathrm{~mm}^{3}$; field of view $=200 \times 200 \times 146 \mathrm{~mm}^{3}$; matrix $=80 \times 78 ; 162$ dynamic scans. During data acquisition, a dark screen (i.e., no visual stimuli) was presented; participants were instructed to remain still, close their eyes and to not think of anything in particular for the duration of the scan ( $5 \mathrm{~min}$ ). High-resolution T1-weighted structural images were acquired with a MPRAGE sequence $(\mathrm{TR} / \mathrm{TE}=9.6 / 4.6 \mathrm{~ms}$; voxel size $=0.98 \times 0.98 \times 1.2$ $\mathrm{mm}^{3}$; field of view $=250 \times 250 \times 228 \mathrm{~mm}^{3} ; 190$ coronal slices). Four participants were scanned with a high-resolution T1-weighted structural MPRAGE sequence with the following parameters: TR/TE $=9.6 / 4.6 \mathrm{~ms}$; voxel size $=0.98 \times 0.98 \times 1.2 \mathrm{~mm}^{3}$; field of view $=250 \times 250 \times 192$ $\mathrm{mm}^{3}$; 160 coronal slices. RS data of each individual were preprocessed as described for the independent RS fMRI dataset (see Supplemental Material). None of the subjects included in the analysis moved more than 1 voxel during the full duration of the scan. The absolute average \pm SD of the maximum displacements across all resting state volumes and 3 planes of movement was $0.39 \pm 0.16 \mathrm{~mm}$ for linear translations and $0.38^{\circ} \pm 0.24^{\circ}$ for rotations. To minimize the impact of excessive motion on the correlations between voxels, volumes in which the scan-to-scan displacement exceeded $0.5 \mathrm{~mm}$ were removed and replaced via interpolation (mean: $0.82 \pm 1.04 \%$, range: $0-3.33 \%$ of acquired volumes discarded). The individual's TBS target was characterized using the same procedure as above but applied at the individual level (i.e., conjunction between the individuals' hippocampus and striatum RSFC maps) and using a 15-mm radius sphere mask centered on the group DLPFC coordinate identified on the independent RS dataset rather than the AAL 
(A) RSFC: HC and Caudate

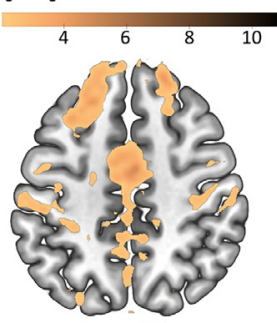

$z=48$

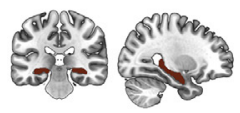

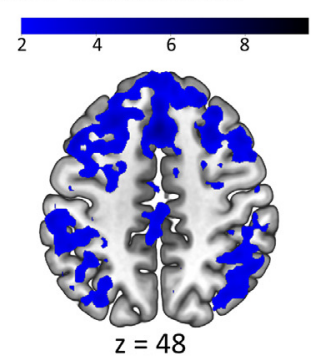

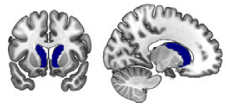

(B) Conjunction map

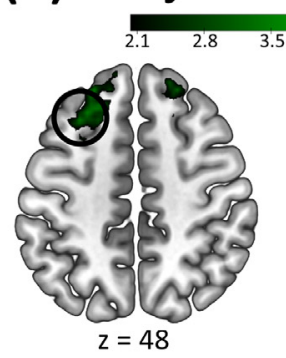

$y=22$

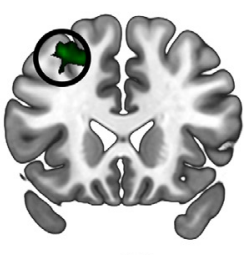

Fig. 2. Group target identification on an independent RS fMRI dataset. (A) Resting State Functional Connectivity (RSFC) maps of the hippocampus (HC, left panel) and the caudate nucleus (right panel). The respective seeds are depicted below the connectivity maps. See Supplemental Table 55 for the complete list of clusters. (B) Conjunction map between the $\mathrm{HC}$ and Caudate RSFC maps (displayed within a frontal mask). A 15-mm radius sphere (depicted as a black circle) centered around the peak maxima (-30 $2248 \mathrm{~mm})$ was used as search area for individualized targeting in the current experiment. See Supplemental Table S6 for a list of prefrontal clusters identified in the conjunction analysis and Supplemental Table S7 and Supplemental Fig. S1 for individual TMS targets of

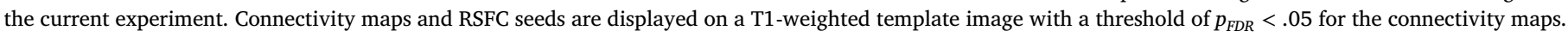
Color bars represent $\mathrm{Z}$ values.

frontal mask for the target search (see Supplemental Table S7 for a list of individual TBS targets and Supplemental Fig. S1 for a depiction of the individual targets).

\subsection{Theta-burst stimulation}

TMS was applied, outside the MRI scanner, with a theta-burst stimulation (TBS) procedure (a burst of 3 pulses given at $50 \mathrm{~Hz}$, repeated every 200 ms; (Huang et al., 2005)) on the individually-identified DLPFC target using a DuoMAG XT-100 rTMS stimulator (DEYMED Diagnostics s.r.o., Hronov, Czech Republic). Online spatial monitoring of the coil position was performed using neuronavigation (BrainSight, Rogue Research Inc, Montreal, Quebec, CA). We applied intermittent (iTBS, $2 \mathrm{~s}$ TBS trains repeated every $10 \mathrm{~s}$ for $190 \mathrm{~s}, 600$ pulses) and continuous TBS (cTBS, $40 \mathrm{~s}$ uninterrupted train of TBS, 600 pulses) at $80 \%$ active motor threshold (MT, (Huang et al., 2005)). Active MT was characterized using single pulse stimulation of the M1 hotspot and motor evoked potentials (MEPs) measured with a belly-tendon EMG montage on the right flexor dorsal interosseous (FDI) muscle. Active MT was probed using a procedure similar to previous reports (Tambini et al., 2018; van Polanen et al., 2020). Specifically, active MT was defined as the lowest intensity at which at least 5 out of 10 MEPs could be distinguished from background EMG during voluntary submaximal FDI contraction. During DLPFC TBS, the $70 \mathrm{~mm}$ DuoMAG butterfly coil was placed at a $45^{\circ}$ angle with the handle pointing posteriorly. Subjects rested for $5 \mathrm{~min}$ post-TBS to not introduce any interfering effects of voluntary movements (Huang et al., 2008). Twenty-one MEPs at $120 \%$ resting MT were measured pre- and 5 min post-TBS (see Fig. 1) as readout of corticospinal excitability (CSE) changes of M1. The first MEP of each time point was discarded from the analyses in each session as its amplitude is usually higher than subsequent MEPs due to startle or reflex responses. Thus, analyses were performed on the remaining $20 \mathrm{MEPs}$, which has been shown to provide a reliable measure of MEP amplitude (Goldsworthy et al., 2016). Resting MT was defined using single pulse stimulation of the M1 hotspot as the lowest intensity at which at least 5 out of 10 MEPs measured on the FDI were larger than $50 \mu \mathrm{V}$. For each participant and within each session, pre-TBS MEPs that were not within the range of the mean \pm 3 SD were excluded $(<1 \%$ of all trials). For each experimental session, post-TBS MEPs were normalized to pre-TBS MEPs and a two-tailed paired t-test ( $\alpha=.05)$ was performed to test for a stimulation effect (cTBS vs. iTBS).

\subsection{Magnetic Resonance Spectroscopy}

\subsubsection{Acquisition}

In-vivo proton $\left({ }^{1} \mathrm{H}\right)$ MRS (Puts and Edden, 2012; Mullins et al., 2014) was used to assess GABA+ levels in the DLPFC TBS target and the hippocampus. Before each MRS acquisition session, a low resolution T1-weighted structural image was acquired for MRS voxel positioning with a MPRAGE sequence $(\mathrm{TR} / \mathrm{TE}=9.6 / 4.6 \mathrm{~ms}$; voxel size $=1.2 \times 1.2 \times 2.0 \mathrm{~mm}^{3}$; field of view $=250 \times 250 \times 222 \mathrm{~mm}^{3}$; 111 coronal slices). Lower- rather than higher-resolution scans were acquired due to time constraints but images showed sufficient quality to position the MRS voxel accurately. For each of the time points (pre-TBS and post-TBS/task) and for each condition, MRS data were acquired using the MEscher-GArwood Point RESolved Spectroscopy (MEGAPRESS) sequence (Mescher et al., 1998) over the individual DLPFC target $\left(30 \times 30 \times 30 \mathrm{~mm}^{3}\right.$ voxel $)$ and the hippocampus $\left(40 \times 25 \times 25 \mathrm{~mm}^{3}\right.$ voxel) with parameters similar to previous research (Hermans et al., 2018; Maes et al., 2018): 320 averages, scan duration of $11 \mathrm{~min}, 14 \mathrm{~ms}$ editing pulses applied at an offset of $1.9 \mathrm{ppm}$ in the ON experiment and $7.46 \mathrm{ppm}$ in the OFF experiment, TR/TE $=2000 / 68 \mathrm{~ms}, 2-\mathrm{kHz}$ spectral width, MOIST water suppression. Sixteen water-unsuppressed averages were acquired at each time point from the same voxel and interleaved to allow for real-time frequency correction (Edden et al., 2016), which is of special importance after fMRI scans (Harris et al., 2014). Scan parameters were identical for all MRS time points.

Before each MRS session, the TBS target was marked for each individual using a fiducial glycerin marker fixated on the participant's head. The specific location on the skull was defined using the nudge tool of the Brainsight software that allows the projection of the individual MNI target coordinate onto the skull. All MRS voxels were positioned according to the MRS time point-specific, low-resolution T1 image. Specifically, the left DLPFC MRS voxel was positioned under this glycerin marker with one surface parallel to the cortical surface in the coronal and sagittal views (see Fig. 3A for an example of voxel positioning and 3B for MRS spectra). The hippocampus voxel was positioned on the coronal view on the center of the left hippocampus and was aligned on the sagittal view parallel to the antero-posterior long axis. Note that we opted to not counterbalance the order of MRS voxel acquisitions and prioritized timing for the DLPFC voxel, as hippocampal MRS data analyses were considered as more exploratory. Therefore, the DLPFC voxel was always acquired before the hippocampus voxel so that the post-TBS/task measurement would be closer in time from the interventions (see Fig. 1). Time constraints prevented us to acquire striatal MRS data as effects of TBS are thought to last on the order of 60 min (Huang et al., 2005). DLPFC and hippocampus voxel placement across sessions and participants are presented in Supplemental Fig. S3. Spatial overlap between sessions and participants was very high for the hippocampus voxel whereas consistency was lower for the DLPFC voxel as placement depended on the individually optimized TBS target.

\subsubsection{Preprocessing and analyses}

The Gannet software 3.0 toolkit (Edden et al., 2014) was used for MRS data analysis similar to previous research in our group 

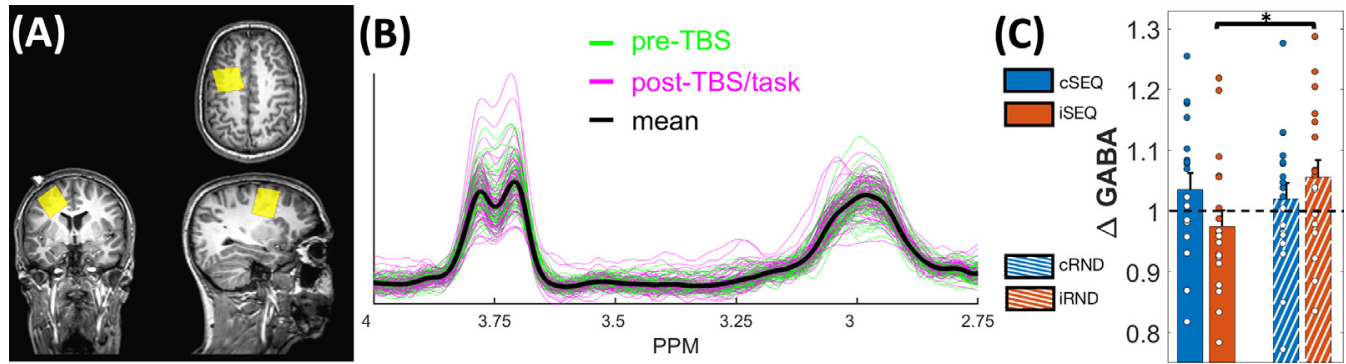

Fig. 3. MRS data of the DLPFC voxel. (A) Depiction of DLPFC MRS voxel positioning of a randomly selected participant and time point. The MRS voxel is overlaid on the participant- and time point-specific T1 structural scan. A glycerin maker was placed at the site of stimulation and was used to optimize MRS voxel positioning (marker visible on the coronal view). See Supplemental Fig. S3 for heatmaps representing the spatial overlap of voxel placement. (B) Spectra of all DLPFC MRS measurements $(N=150)$, from all participants and time points. GABA+ peak is visible at $3 \mathrm{ppm}$. Pre-TBS and post-TBS/task time points are depicted in green and magenta, respectively (mean spectrum across all participants and time points depicted in black). (C) $\triangle$ GABA in the four experimental conditions. Note that a preto post-intervention GABA+ increase and decrease are represented by values above and below 1 (indicated by the black dashed line), respectively. Exploratory analyses indicate that $\triangle$ GABA significantly differed between the iSEQ and iRND conditions. See Supplemental Table S8 for quality metrics and tissue segmentation, Supplemental Table S9 for follow-up tests for significant effects on tissue fractions and Supplemental Table S10 for raw GABA+ values. Error bars indicate SEM. Circles represent individual data points. Asterisk represents significant paired $t$-test with $p<.05$ (uncorrected for multiple comparisons). TBS: theta-burst stimulation, i: intermittent, c: continuous, SEQ: sequence, RND: random.

(Hermans et al., 2018; Maes et al., 2018; King et al., 2020). We corrected the individual frequency-domain spectra for frequency and phase using spectral registration in the time domain (Near et al., 2015). A $3 \mathrm{~Hz}$ exponential line broadening filter was applied subsequently. An edited difference spectrum was derived from the averaging and subtracting of individual ON and OFF spectra. The GABA signal from this difference spectrum was modelled at $3 \mathrm{ppm}$ with a single Gaussian peak and a 5-parameter Gaussian model using the combined GABAGlx model. A Gaussian-Lorentzian model was used to fit the unsuppressed water signal that was used as the reference compound (Mikkelsen et al., 2019). Uncorrected GABA levels were quantified from the integrals of the modelled data. It is worth noting that this approach edits GABA as well as macromolecules at 3 ppm (Rothman et al., 1993; Edden et al., 2012) and thus GABA levels are reported as GABA+ (GABA plus macromolecules). The high-resolution T1-weighted image acquired during baseline was co-registered to the 8 ( 2 pre- and post-intervention time points $\mathrm{x} 4$ conditions) low-resolution images using SPM12, so that the high-resolution structural image could be used for data processing for each MRS time point in each condition. MRS voxels were co-registered to the highresolution T1-weighted image and were segmented into different tissue fractions (gray matter [GM], white matter [WM], and cerebrospinal fluid [CSF]) to adjust GABA+ levels for heterogeneity in voxel tissue composition. It was assumed that GABA+ levels are negligible in CSF and twice as high in GM relative to WM (Harris et al., 2015) to compute tissue-corrected GABA+. Tissue-specific relaxation as well as water visibility values were also considered (Harris et al., 2015). Last, GABA+ levels were normalized to the average voxel composition in the sample (Harris et al., 2015). Therefore, the reported GABA+ values correspond to the "QuantNormTissCorrGABAiu" variable in Gannet 3.0, specified in institutional units [i.u.].

Due to low hippocampal MRS data quality, presumably due to difficulties associated with shimming in deep brain regions and participant movement between the low-resolution T1 (measured just before the RS, see Fig. 1) and the hippocampal MRS scans, the fitting step as part of the Gannet pipeline failed in 15 out of 150 measurements during preprocessing. This resulted in 12 missing conditions, with a complete condition consisting of both the pre and post MRS time points for that particular experimental session ( 6 participants with 1 condition missing and 3 participants with 2 conditions missing). As too few measurements were left for appropriate statistical analyses of the hippocampal MRS data (only 10 participants with complete data sets), MRS analyses presented in this paper were limited to the DLPFC voxel.

Quality of the DLPFC MRS data was assessed by examining GABA signal-to-noise (SNR) ratio, fit error, and frequency offset. MRS voxel tissue fractions, quality metrics and corresponding statistical analyses to assess potential effects of MRS time point and experimental condition can be found in Supplemental Table S8 and S9 and in the Supplemental Results.

For each experimental session, post-TBS/task GABA+ levels were normalized to pre-TBS GABA+ levels $\left(\mathrm{GABA}+{ }_{\text {pre }} / \mathrm{GABA}+{ }_{\text {post }}\right.$, referred to as $\triangle \mathrm{GABA}$, see Supplemental Table S10 for raw data) and the data were analyzed using repeated measures analyses of variance (ANOVAs; $\alpha=.05$ ) with stimulation (cTBS and iTBS) and task (SEQ and RND) as within-subject factors. Exploratory follow-up two-tailed paired t-tests ( $\alpha=.05$ ) were performed on all possible pairs. Additional control analyses were performed on the $\triangle \mathrm{GABA}$ with linear mixed models taking into account visit effects (see Supplemental Results). The individual normalized GABA+ data $(\triangle \mathrm{GABA})$ of each condition were also used as covariates for fMRI regression analyses (see details below).

\subsection{Task-related fMRI data acquisition and analysis}

\subsubsection{Acquisition}

Task-related fMRI data were acquired using an ascending gradient EPI pulse sequence for T2 $*$-weighted images $(\mathrm{TR}=2000 \mathrm{~ms}$; TE $=29.8$ ms; multiband factor 2; flip angle $=90^{\circ}$; 54 transverse slices; slice thickness $=2.5 \mathrm{~mm}$; interslice gap $=0.2 \mathrm{~mm}$; voxel size $=2.5 \times 2.5 \times 2.5$ $\mathrm{mm}^{3}$; field of view $=210 \times 210 \times 145.6 \mathrm{~mm}^{3}$; matrix $=84 \times 82 ; 345.09$ \pm 22.37 dynamical scans).

\subsubsection{Spatial pre-processing}

Task-based functional volumes of each participant were realigned to the first image of each session and then realigned to the across-session mean functional image using rigid body transformations. The mean functional image was co-registered to the high-resolution T1-weighted anatomical image using a rigid body transformation optimized to maximize the normalized mutual information between the two images. The resulting co-registration parameters were then applied to the realigned functional images. The structural image was segmented into gray matter, white matter, cerebrospinal fluid (CSF), bone, soft tissue, and background. We created an average subject-based template using DARTEL in SPM12, registered to the Montreal Neurological Institute (MNI) space. All functional and anatomical images were then normalized to the resulting template. Functional images were spatially smoothed using an isotropic $8 \mathrm{~mm}$ full-width at half-maximum (FWHM) Gaussian kernel.

\subsubsection{Activation analyses}

The analysis of task-based fMRI data, based on a summary statistics approach, was conducted in 2 serial steps accounting for intra-individual 
(fixed effects) and inter-individual (random effects) variance, respectively. Changes in brain regional responses were estimated for each participant with a model including responses to the motor task and its linear modulation by performance speed (mean RT on correct button presses per block) in each session (cSEQ, cRND, iSEQ and iRND). Performance speed, rather than accuracy, was chosen as a parametric modulator because performance accuracy remained stable during practice (see results section) and was therefore not modulated by task practice. These regressors consisted of box cars convolved with the canonical hemodynamic response function. The 15-second rest blocks occurring between each block of motor practice served as the baseline condition modeled implicitly in the block design. Movement parameters derived from realignment as well as erroneous key presses were included as covariates of no interest. Movements were minimal during scanning; only the data of one session in one participant were excluded for excessive movement ( $>2$ voxels; note that for another participant, the last 46 scans of one session were excluded from analyses because of movements but the truncated session was kept in the analyses). The average \pm SD translation and rotation across axis and sessions was: $1.07 \pm 0.62 \mathrm{~mm}$ and 1.10 $\pm 0.61^{\circ}$ (maximum absolute movement in translation $=3.7 \mathrm{~mm}$ and in rotation $=2.9^{\circ}$ ). High-pass filtering was implemented in the design matrix using a cutoff period of $128 \mathrm{~s}$ to remove slow drifts from the time series. Serial correlations in the fMRI signal were estimated using an autoregressive (order 1 ) plus white noise model and a restricted maximum likelihood (ReML) algorithm.

Linear contrasts tested the main effect of practice and its linear modulation by performance speed in each session as well as between sessions. Contrasts testing for the stimulation by task interaction [(iTBS vs. cTBS) x (SEQ vs. RND)] and the stimulation effect within each task condition [iSEQ vs. cSEQ] and [iRND vs. cRND] were generated at the individual level. To examine whether the dynamics of brain responses were influenced by stimulation conditions, contrasts tested for the stimulation effect on the modulation regressors. As performance levels remained - as expected - constant in the random conditions (see results), this set of analyses focused on the sequence conditions only [iSEQ $Q_{\bmod }$ vs. $\mathrm{cSEQ}_{\mathrm{mod}}$. Additional contrasts presented in the Supplemental Information tested for the modulation effect across stimulation conditions within the sequence task $\left[\mathrm{iSEQ}_{\mathrm{mod}}+\mathrm{cSEQ} \mathrm{Q}_{\mathrm{mod}}\right.$ ] (see Supplemental Table S11). The resulting contrast images were further spatially smoothed (Gaussian kernel $6 \mathrm{~mm}$ FWHM) and were entered in a second level analysis for statistical inference at the group level (one sample t-tests), corresponding to a random effects model accounting for inter-subject variance.

To assess the relationship between any effect highlighted in the contrasts described above and the pre- to post-intervention changes in $\mathrm{GABA}+$ levels (referred to as $\triangle \mathrm{GABA}$ ), we performed regression analyses at the second level using one sample t-test with multiple covariates. Specifically, we regressed the individual contrast images testing for the stimulation by task interaction [(iSEQ - iRND) - (cSEQ - cRND)] against individual $\triangle$ GABA measured in the four conditions (4 covariates). The multiple regression therefore tested whether stimulation by task-related activity patterns correlated with stimulation by task-related changes in GABA levels in the DLPFC $\left[\left(\Delta \mathrm{GABA}_{\mathrm{iSEQ}}-\Delta \mathrm{GABA}_{\mathrm{iRND}}\right)-\left(\Delta \mathrm{GABA}_{\mathrm{cSEQ}}\right.\right.$ - $\left.\left.\triangle \mathrm{GABA}_{\mathrm{cRND}}\right)\right]$. A separate multiple regression analysis tested whether the stimulation effect on dynamical activity within the SEQ task condition [iSEQ ${ }_{\text {mod }}$ vs. $c S E Q_{\text {mod }}$ ] correlated with the stimulation effect on $\triangle \mathrm{GABA}$ in the corresponding conditions $\left[\triangle \mathrm{GABA}_{\mathrm{iSEQ}} \mathrm{vs.} \triangle \mathrm{GABA}_{\mathrm{CSEQ}}\right]$. In these regression analyses, any significant brain response is differently related to $\triangle \mathrm{GABA}$ between stimulation (or stimulation by task; for the interaction contrast) conditions.

\subsubsection{Functional connectivity analyses}

Psychophysiological interaction (PPI) analyses were computed to test the functional connectivity of the individual DLPFC targets and subcortical a priori regions of interest (i.e. the striatum and the hippocampus) highlighted by the activation-based contrasts. Seed coordinates for the DLPFC connectivity analyses consisted of the individual TBS targets as identified with the RS pipeline (see above). Note that the group, rather than the individual, target was used in two participants as their individual coordinates were located close to the cortex's edge which did not allow the extraction of enough seed signal (see procedure below). Two putamen, but no hippocampal, seed regions were identified based on activation analyses. PPI analyses were performed using the peak coordinate of the two significant putamen clusters highlighted in the group level activation maps (iSEQ ${ }_{\text {mod }}+{ }_{c S E Q}$ mod, see Supplemental Table S11: [24 12 l $4 \mathrm{~mm}$ ] and [-16 6 -6 mm]). For each participant, experimental session and seed region of interest, the first eigenvariate of the signal was extracted using Singular Value Decomposition of the time series across the voxels included in a $10 \mathrm{~mm}$ radius sphere centered around the seed of interest. A new linear model was generated at the individual level, using three regressors for each experimental session. The first regressor corresponded to the BOLD activity in the reference area. The second regressor represented the practice of the learned sequence or the practice of the learned sequence modulated by performance speed. The third regressor represented the interaction of interest between the first (physiological) and the second (psychological) regressors. To build this regressor, the underlying neuronal activity was first estimated by a parametric empirical Bayes formulation, combined with the psychological factor, and subsequently convolved with the hemodynamic response function (Gitelman et al., 2003). The design matrix also included movement parameters. A significant PPI indicated a change in the regression coefficients (i.e. a change in the strength of the functional interaction) between any reported brain area and the reference region, related to the practice of the task or to the change in performance speed during the practice of the task. Linear contrasts testing the stimulation by task interaction [(iTBS vs. cTBS) x (SEQ vs. RND)] as well as the main effect of stimulation on modulation within SEQ conditions [iSEQ $_{\text {mod }}$ vs. $\left.c S E Q_{\text {mod }}\right]$ were generated at the individual level. The resulting contrast images were further spatially smoothed (Gaussian kernel 6 mm FWHM) and were entered in a second level analysis for statistical inference at the group level (one sample t-tests), corresponding to a random effects model accounting for inter-subject variance. Furthermore, we assessed the relationship between DLPFC connectivity patterns and $\triangle$ GABA levels in the DLPFC with regression analyses at the second level using one sample t-test with multiple covariates. As no significant responses were observed for the DLPFC connectivity analyses on the interaction contrast (see results), regression analyses were only performed on the DLPFC PPI analyses testing for the stimulation effect within SEQ conditions. Specifically, we regressed the individual contrast images testing for the difference in dynamical connectivity between the two SEQ conditions [iSEQ ${ }_{\text {mod }}$ vs. cSEQ ${ }_{\text {mod }}$ ] against the $\triangle \mathrm{GABA}$ in these two conditions $\left[\triangle \mathrm{GABA}_{\mathrm{iSEQ}}\right.$ vs. $\left.\triangle \mathrm{GABA}_{\mathrm{cSEQ}}\right]$. In these analyses, any significant brain response shows connectivity patterns with the DLPFC during sequence learning that are differently related to the change in DLPFC GABA between stimulation conditions.

In order to control for potential confounds due to the post-processing unbalancing in conditions per visit (see Supplemental Table S4), the activity and connectivity second-level analyses described above were repeated using visit as a covariate of no-interest coded with dummy variables (see Supplemental Results).

\subsubsection{Statistical inferences}

The set of voxel values resulting from each analysis described above (activation and functional connectivity) constituted maps of the t statistics $[\mathrm{SPM}(\mathrm{T})]$, thresholded at $p<.005$ (uncorrected for multiple comparisons). Statistical inferences were performed on a priori defined ROIs including the DLPFC search sphere, the hippocampi and the basal ganglia (putamen, caudate nucleus and globus pallidus), at a threshold of $p<.05$ after family-wise error (FWE) correction for multiple comparisons over small volume within the ROIs (small volume correction (SVC) approach; (Poldrack, 2007; Poldrack et al., 2008)), followed by Holm-Bonferroni correction to correct for multiple ROI testing within each contrast ( $p$ 


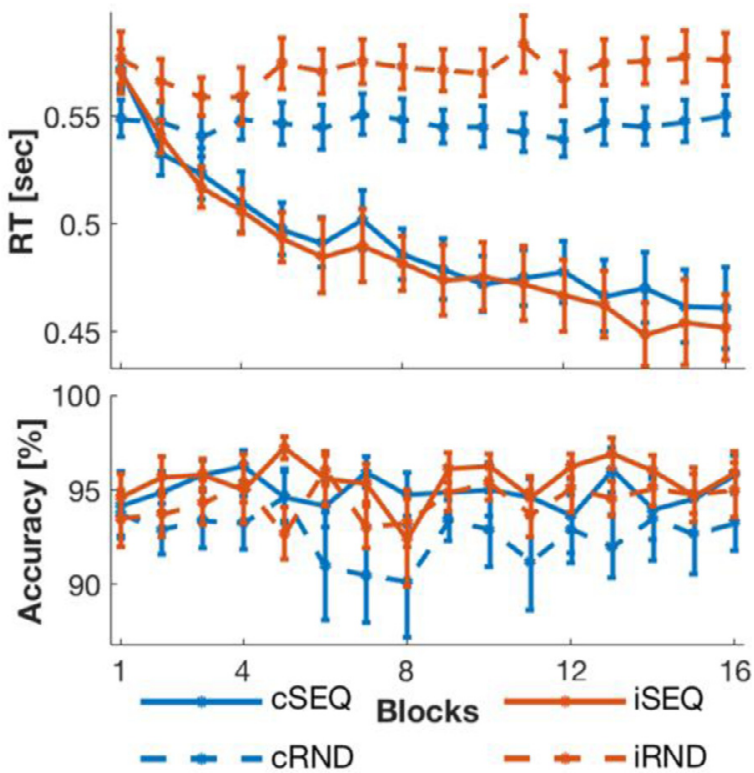

Fig. 4. Behavioral results. Upper panel: Performance speed (reaction time, RT) improved over the course of training in the sequence task (SEQ) conditions and stayed stable in random task (RND) conditions. Lower panel: Performance accuracy remained stable in all conditions with overall higher accuracy in the SEQ than in the RND condition. The stimulation intervention (c: continuous and $\mathrm{i}$ : intermittent) did not affect motor performance nor motor learning.

$<$.05) (Holm, 1979). For SVC, spheres (10 mm radius) were centered on coordinates of our ROIs taken from the literature (see Supplemental Table S12). All reported activations in the main text survived SVC and Holm-Bonferroni correction for multiple comparisons on the contrast level. For the sake of completeness, results of whole brain analyses are reported in Supplemental Tables S11, S13, S14, S15 and Supplemental Results.

\section{Results}

Results related to corticospinal excitability, sleep and vigilance can be found in the Supplemental Information. In summary, corticospinal excitability was not modulated by the different stimulation conditions. There were no differences in sleep quantity and quality between experimental sessions and the differences in subjective vigilance observed between sessions did not influence behavior (see Supplemental Results for details).

\subsection{Behavior}

Performance speed across the 16 blocks of task practice was faster during the SEQ as compared to the RND task condition (main effect of task; $\mathrm{F}_{(1,16)}=40.435, \eta_{\mathrm{p}}^{2}=.716, p<.001$ ) and improved over the course of training across task conditions (main effect of block; $\left.\mathrm{F}_{(3.419,54.7)}=16.325, \mathrm{\eta}_{\mathrm{p}}{ }^{2}=.505, p<.001\right)$. This increase was more pronounced in the SEQ as compared to the RND task (task by block interaction; $\mathrm{F}_{(3.838,61.415)}=21.492, \mathrm{\eta}_{\mathrm{p}}{ }^{2}=.573, p<.001$; Fig. 4, upper panel). No effects of stimulation $\left(\mathrm{F}_{(1,16)}=1.639, \mathrm{\eta}_{\mathrm{p}}{ }^{2}=.093, p=.219\right)$, stimulation by task $\left(\mathrm{F}_{(1,16)}=2.102, \eta_{\mathrm{p}}^{2}=.116, p=.166\right)$, stimulation by block $\left(\mathrm{F}_{(7.396,118.341)}=.446, \mathrm{\eta}_{\mathrm{p}}{ }^{2}=.027, p=.88\right)$ or stimulation by task by block $\left(\mathrm{F}_{(6.155,98.477)}=.566, \mathrm{\eta}_{\mathrm{p}}{ }^{2}=.034, p=.76\right)$ were observed for performance speed (but see Supplemental Information for exploratory analyses on the effect of stimulation within the random task).

Performance accuracy was higher during SEQ compared to RND practice (main effect of task; $\mathrm{F}_{(1,16)}=6.919, \mathrm{\eta}_{\mathrm{p}}{ }^{2}=.302, p=.018$; Fig. 4, lower panel). No effects of stimulation $\left(\mathrm{F}_{(1,16)}=2.367, \mathrm{\eta}_{\mathrm{p}}{ }^{2}=.129\right.$, $p=.143)$, block $\left(\mathrm{F}_{(4.815,77.033)}=1.552, \eta_{\mathrm{p}}{ }^{2}=.088, p=.186\right)$, stimulation by task $\left(\mathrm{F}_{(1,16)}=.31, \mathrm{\eta}_{\mathrm{p}}^{2}=.019, p=.585\right)$, stimulation by block $\left(\mathrm{F}_{(5.635,90.163)}=.662, \eta_{\mathrm{p}}^{2}=.04, p=.671\right)$, task by block $\left(\mathrm{F}_{(15,240)}=.643, \eta_{\mathrm{p}}{ }^{2}=.039, p=.837\right)$ or stimulation by task by block $\left(\mathrm{F}_{(3.476,55.619)}=.759, \mathrm{\eta}_{\mathrm{p}}^{2}=.045, p=.54\right)$ were observed for performance accuracy.

Results of control analyses modelling the visit effect were similar to those reported above for both performance speed and accuracy (see Supplemental Results for details).

Altogether, the behavioral results demonstrated that participants learned the motor sequence and that the stimulation intervention did not impact motor sequence learning nor overall motor performance.

\subsection{MRS of GABA}

Fitting of the GABA peak failed in a high proportion of measurements for the hippocampal MRS data, leaving only 10 complete data sets (see methods for further information). As too few measurements remained for appropriate statistical analyses of the hippocampal MRS data, results presented in this paper are limited to the DLPFC voxel (see Fig. 3A for a depiction of DLPFC MRS voxel positioning and Supplemental Fig. S3 for voxel placements across sessions and participants).

Post-TBS/task GABA+ levels were normalized to pre-TBS GABA+ levels in order to assess intervention-related GABA+ changes (referred to as $\triangle$ GABA, see Supplemental Table S10 for raw data and Fig. 3B for spectra of all DLPFC MRS measurements). $\triangle$ GABA was not significantly influenced by the task $\left(\mathrm{F}_{(1,16)}=2.181, \mathrm{\eta}_{\mathrm{p}}{ }^{2}=.12, p=.159\right)$, stimulation $\left(\mathrm{F}_{(1,16)}=.025, \mathrm{\eta}_{\mathrm{p}}{ }^{2}=.002, p=.876\right)$ or by an interaction between task and stimulation $\left(\mathrm{F}_{(1,16)}=2.975, \mathrm{\eta}_{\mathrm{p}}^{2}=.157, p=.104\right)$. However, exploratory paired t-tests indicated that GABA+ levels were significantly reduced after sequence learning as compared to random practice under the influence of iTBS (iSEQ vs. iRND; $\mathrm{t}_{(1,17)}=-2.508, \mathrm{~d}_{\mathrm{z}}=-.59$, $p=.023$ (uncorrected for multiple comparisons; Fig. 3C). None of the other paired comparisons were significant (all $p s>.05$ ). Results of control analyses modelling the visit effect were similar to those reported above (see Supplemental Results for details).

\subsection{Functional brain imaging data}

Using an ROI approach including the basal ganglia, the hippocampus and the DLPFC TMS target, we investigated the effects of stimulation and task conditions on the amplitude and dynamics of task-related activity and connectivity. Additionally, we performed regression analyses between $\triangle$ GABA and the above-mentioned activity and DLPFC connectivity maps to assess the relationships between changes in prefrontal GABA pre- to post-intervention and BOLD responses during task performance.

\subsubsection{Stimulation by task interaction}

Results show that stimulation and task conditions did not interact with brain activity or connectivity patterns in our ROIs and did not modulate the relationship between BOLD signal in the ROIs and DLPFC GABA changes (but see Supplemental Table S13, Supplemental Fig. S4 and Supplemental Results for results of the whole brain analyses showing stimulation by task interaction effects in the intraparietal sulcus, the cerebellar lobule and the frontal cortex). To mirror the exploratory analyses on GABA+ levels described above, we tested whether the responses between iSEQ and iRND and the corresponding $\triangle$ GABA were related but did not observe any significant responses in the ROIs (see Supplemental Table S13).

\subsubsection{Learning-related modulation of brain responses}

We used parametric modulation analyses to test whether brain activity changed as a function of learning, i.e. the block-to-block performance improvements, in the SEQ conditions. This allowed us to examine whether the different stimulation conditions influenced the learningrelated dynamics of brain responses. 
Table 2

Functional imaging results for the main effect of stimulation on brain responses modulated by performance speed during sequence learning.

\begin{tabular}{|c|c|c|c|c|c|c|}
\hline Area & $\mathrm{x} \mathrm{mm}$ & y mm & $\mathrm{z} \mathrm{mm}$ & $\mathrm{k}$ voxels & $\mathrm{T}$ & $p_{\text {FWEsvc }}$ \\
\hline \multicolumn{7}{|l|}{ Activation } \\
\hline \multirow{2}{*}{\multicolumn{7}{|c|}{$\begin{array}{l}\mathrm{cSEQ}_{\text {mod }}-\mathrm{iSEQ}_{\text {mod }} \\
\text { No significant responses }\end{array}$}} \\
\hline & & & & & & \\
\hline \multicolumn{7}{|c|}{$\mathrm{iSEQ}_{\bmod }-\mathrm{CSEQ}_{\bmod }$} \\
\hline \multicolumn{7}{|c|}{ No significant responses in the ROIs } \\
\hline \multicolumn{7}{|c|}{ Regression with $\triangle \mathrm{GABA}$} \\
\hline \multicolumn{7}{|c|}{$\left(\mathrm{iSEQ}_{\bmod }-\mathrm{CSEQ}_{\mathrm{mod}}\right) \times\left(\triangle \mathrm{GABA}_{\mathrm{iSEQ}}-\Delta \mathrm{GABA}_{\mathrm{CSEQ}}\right)$} \\
\hline Hippocampus & 30 & -16 & -18 & 159 & 4.6 & .009 \\
\hline \multicolumn{7}{|c|}{$\left(\mathrm{iSEQ}_{\bmod }-\mathrm{CSEQ}_{\mathrm{mod}}\right) \times\left(\triangle \mathrm{GABA}_{\mathrm{CSEQ}}-\Delta \mathrm{GABA}_{\mathrm{iSEQ}}\right)$} \\
\hline Putamen & -22 & 2 & -2 & 1256 & 5.82 & .002 \\
\hline \multicolumn{7}{|c|}{ Right putamen connectivity } \\
\hline Caudate & 10 & 12 & -8 & 24 & 3.62 & .033 \\
\hline \multicolumn{7}{|l|}{$\mathrm{iSEQ}_{\bmod }-\mathrm{CSEQ}_{\bmod }$} \\
\hline \multirow[t]{2}{*}{ Putamen } & 28 & -8 & -2 & 125 & 3.99 & .019 \\
\hline & -18 & 4 & -4 & 24 & 3.37 & .048 \\
\hline \multicolumn{7}{|c|}{ Left putamen connectivity } \\
\hline \multicolumn{7}{|c|}{$\mathrm{cSEQ}_{\text {mod }}-\mathrm{iSEQ}_{\text {mod }}$} \\
\hline $\begin{array}{l}\mathrm{iSEQ}_{\bmod }-\mathrm{CSEQ}_{\bmod } \\
\text { Putamen }\end{array}$ & 28 & 8 & 16 & 403 & 5.01 & .003 \\
\hline \multicolumn{7}{|l|}{ DLPFC connectivity } \\
\hline \multicolumn{7}{|l|}{$\begin{array}{l}\mathrm{CSEQ}_{\text {mod }}-\mathrm{iSEQ}_{\text {mod }} \\
\text { No significant responses }\end{array}$} \\
\hline \multicolumn{7}{|l|}{$\begin{array}{l}\text { No signincant responses } \\
\text { iSEQ } \text { mod }^{-} \mathrm{CSEQ}_{\bmod }\end{array}$} \\
\hline Posterior hippocampus & 22 & -40 & 0 & 42 & 3.85 & .024 \\
\hline \multicolumn{7}{|c|}{ Regression between DLPFC connectivity and $\triangle \mathrm{GABA}$} \\
\hline \multicolumn{7}{|c|}{$\left(\mathrm{iSEQ}_{\bmod }-\mathrm{CSEQ}_{\bmod }\right) \times\left(\Delta \mathrm{GABA}_{\mathrm{iSEQ}}-\Delta \mathrm{GABA}_{\mathrm{cSEQ}}\right)$} \\
\hline DLPFC & -30 & 24 & 50 & 69 & 3.72 & .036 \\
\hline \multicolumn{7}{|c|}{$\left(\mathrm{iSEQ}_{\bmod }-\mathrm{CSEQ}_{\bmod }\right) \times\left(\Delta \mathrm{GABA}_{\mathrm{cSEQ}}-\Delta \mathrm{GABA}_{\mathrm{iSEQ}}\right)$} \\
\hline \multirow[t]{2}{*}{ Putamen } & 20 & 4 & -6 & 829 & 4.61 & .01 \\
\hline & 30 & -10 & 0 & 73 & 4.44 & .013 \\
\hline Hippocampus & -24 & -20 & -12 & 1031 & 4.77 & .008 \\
\hline
\end{tabular}

Brain responses significant $\left(p_{\text {corr }}<.05\right)$ after family-wise error (FWE) correction for multiple comparisons over a small volume of interest (svc) in the ROIs are reported here. All activations survive Holm-Bonferroni correction for multiple comparisons within each contrast. Voxels of these maps not surviving correction for multiple comparisons and which were not of interest were not reported. See Supplemental Table S11 for modulation of brain responses by performance speed during practice of the sequential motor task (iSEQ ${ }_{\text {mod }}+\mathrm{cSEQ}_{\text {mod }}$ ), Supplemental Table S12 for coordinates of areas of interest used for spherical small volume corrections, Supplemental Table S13 for whole brain functional imaging results for the stimulation by task interaction contrasts, and Supplemental Table S14 for whole brain functional imaging results for the main effect of stimulation on brain responses modulated by performance speed during sequence learning.

SEQ $=$ sequence, $\mathrm{i}=$ intermittent, $\mathrm{c}=$ continuous, $\bmod =$ modulation contrast, $\mathrm{GABA}=$ gamma-aminobutyric acid, DLPFC $=$ dorsolateral prefrontal cortex.

\subsection{Activity}

Consistent with previous research (Albouy et al., 2012), activity in bilateral putamen increased as a function of learning regardless of the type of stimulation (i.e., iSEQ ${ }_{\text {mod }}+\mathrm{cSEQ}_{\text {mod }}$, Supplemental Table S11). Between-stimulation-condition contrasts showed no significant results within the ROIs (but see Supplemental Table S14 for results of the whole brain analyses showing between-stimulation-condition effects in superior frontal areas, central sulcus and cingulum).

We then conducted regression analyses assessing whether betweencondition differences in dynamical brain activity during training were related to differences in DLPFC $\triangle$ GABA between conditions. Re-

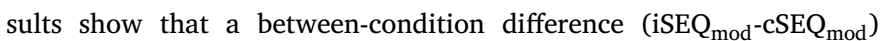
in dynamical activity in the hippocampus was related to the difference in DLPFC $\triangle \mathrm{GABA}$ between stimulation conditions $\left(\triangle \mathrm{GABA}_{\mathrm{iSEQ}^{-}}\right.$ $\triangle$ GABA $_{\text {cSEO }}$, Table 2; Fig. 5A). Interestingly, between-condition differences in dynamical activity in putamen activity were also related to the difference in DLPFC $\triangle$ GABA between stimulation conditions, but in the opposite direction ( $\triangle \mathrm{GABA}_{\mathrm{CSEQ}}-\triangle \mathrm{GABA}_{\mathrm{iSEQ}}$, Table 2$)$ as compared to the hippocampus. The effects reported above were similar when controlling for the visit effect (see Supplemental Table S16).

These results collectively indicate that the DLPFC stimulation conditions differently influenced the relationship between changes in DLPFC GABA levels and learning-related changes in activity patterns in the hippocampus and the striatum.

\subsection{Connectivity}

Connectivity analyses were performed using, as seed regions, the putamen clusters described above that exhibited increases in activity as a function of learning across the two stimulation conditions (Supplemental Table S11). Functional connectivity between these bilateral putamen seeds and sensorimotor parts of the putamen increased as a function of learning more in the iSEQ as compared to the cSEQ condition (iSEQ $\bmod ^{-}$ $\mathrm{cSEQ}_{\text {mod }}$, Table 2; Fig. 6A upper panel). In contrast, the right putamen showed a greater learning-related increase in connectivity with the caudate nucleus, a more associative territory of the striatum, in the cSEQ 

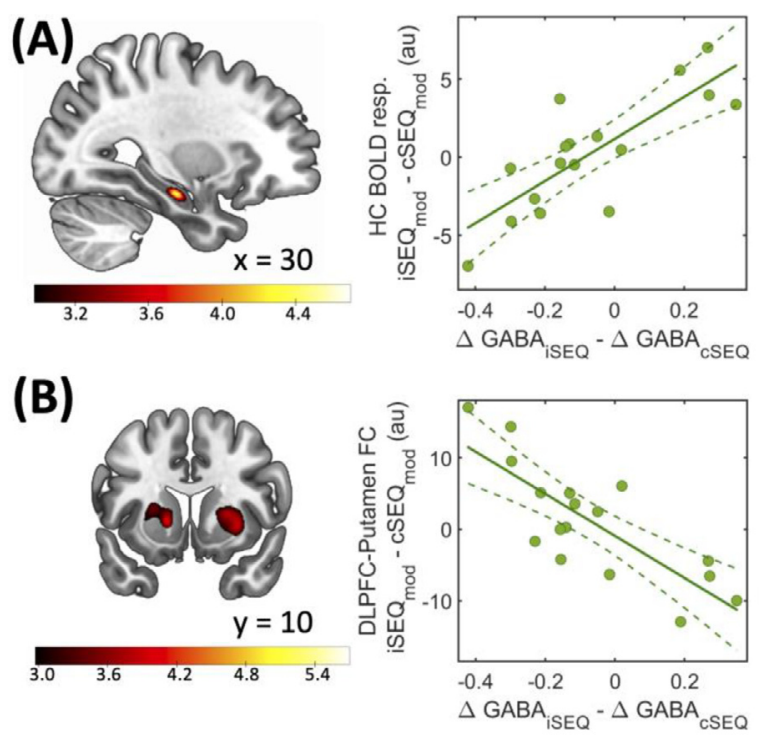

Fig. 5. Regressions with DLPFC $\triangle$ GABA. (A) Hippocampal (HC) dynamical activity during learning (30-16 $-18 \mathrm{~mm}$, left panel) was differently related to DLPFC $\triangle$ GABA between conditions. (B) Learning-related changes in DLPFCputamen functional connectivity (FC) patterns (20 $4-6 \mathrm{~mm}$, left panel) were differently related to DLPFC $\triangle$ GABA between conditions. Regression maps are displayed on a T1-weighted template image with a threshold of $p<.005$ uncorrected. Color bars represent $\mathrm{T}$ values. Circles represent individual data, solid lines represent linear regression fits, dashed lines depict 95\% prediction intervals of the linear function. au: arbitrary units, resp.: response, i: intermittent, c: continuous, SEQ: sequence, mod: modulation contrast, GABA = gammaaminobutyric acid. as compared to the iSEQ condition (cSEQ mod $-\mathrm{iSEQ}_{\text {mod }}$, Table 2; Fig. 6A lower panel).

Functional connectivity analyses using the DLPFC TMS target as a seed region indicate that the dynamical connectivity patterns between the DLPFC and the hippocampus were different between stimulation conditions. These differences in fronto-hippocampal connectivity were explained by antagonistic dynamical patterns between conditions; specifically, connectivity decreased and increased as a function of learning in the iTBS and cTBS conditions, respectively (iSEQ mod $^{-} \mathrm{CSEQ}_{\mathrm{mod}}$ contrast, Table 2; Fig. 6B; and see Supplemental Table S14 for whole brain analyses).

Regression analyses linking between-condition differences in DLPFC connectivity (iSEQ mod $-\mathrm{cSEQ}_{\text {mod }}$ ) to $\triangle \mathrm{GABA}\left(\triangle \mathrm{GABA}_{\mathrm{iSEQ}}\right.$ vs. $\triangle \mathrm{GABA}_{\mathrm{CSEQ}}$ ) showed that the dynamical connectivity patterns between the DLPFC and the putamen as well as the hippocampus were differently related to the DLPFC $\triangle$ GABA between stimulation conditions (Table 2, Fig. 5B; and see Supplemental Table S14 for whole brain analyses showing additional cerebellar and parietal regions).

All the functional connectivity results reported above were similar when controlling for the visit effect (see Supplemental Table S16).

Altogether, our results indicate that iTBS, as compared to cTBS, applied to the DLPFC before motor sequence learning promoted learningrelated increases in connectivity in sensorimotor-striatal networks. In contrast, cTBS of the DLPFC resulted in progressive connectivity increases in fronto-hippocampal and associative-striatal networks. Additionally, our findings show that the stimulation conditions differently altered the relationship between the learning-related changes in DLPFCstriatum-hippocampus connectivity and DLPFC $\triangle$ GABA.

\section{Discussion}

In this proof-of-concept study, we used a multimodal neuroimaging approach in order to investigate whether functional-data-driven prefrontal stimulation tailored to each individual can alter neural responses

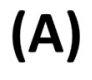

(A)

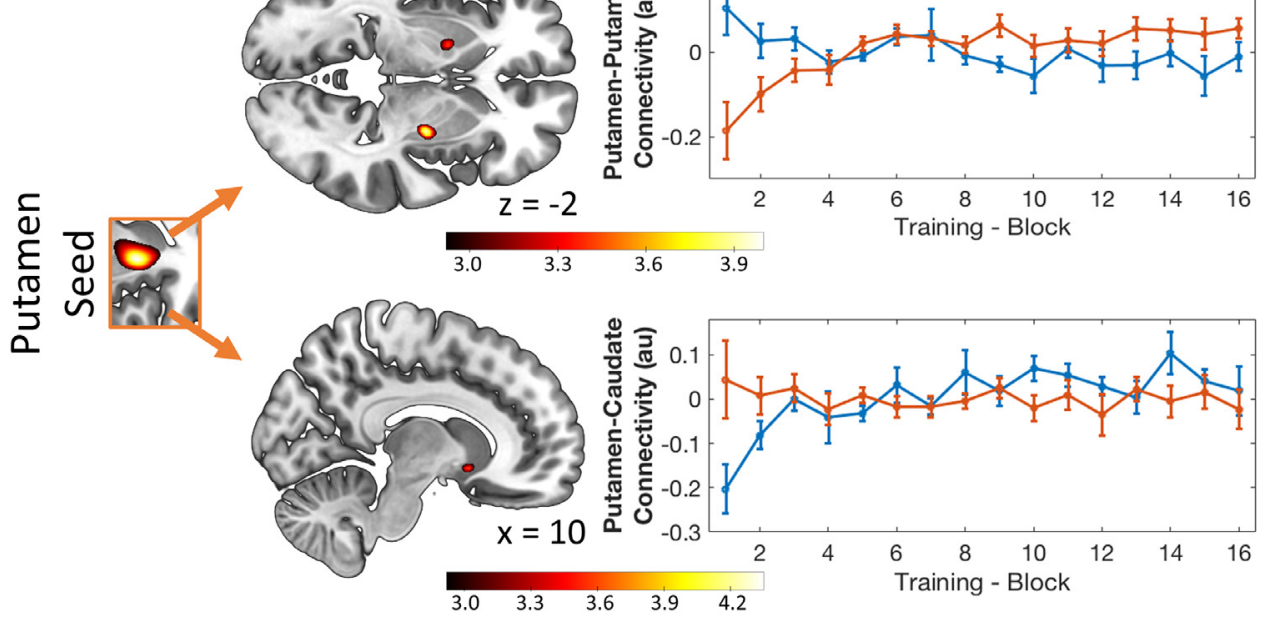

(B)

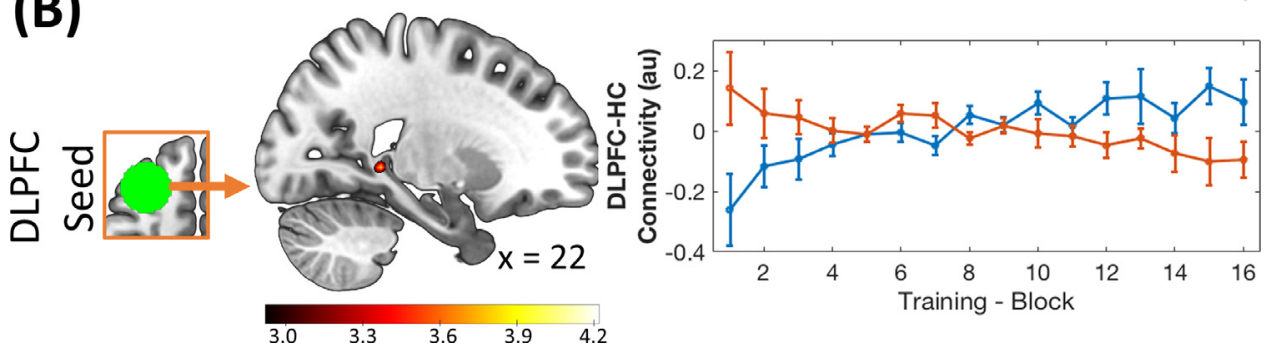

Fig. 6. Stimulation effect on sequence (SEQ) task-related connectivity. (A) Functional connectivity (FC) between the right putamen and the sensorimotor putamen (28 -8 -2 mm, upper panel) increased more as a function of learning after iTBS compared to cTBS. FC with the caudate nucleus (10 12 -8 mm, lower panel) showed the opposite pattern. (B) FC of the DLPFC TBS target with the hippocampus (HC, $22-400 \mathrm{~mm}$ ) increased more as a function of learning in the cTBS as compared to the iTBS condition. Connectivity maps are displayed on a T1-weighted template image with a threshold of $p<.005$ uncorrected. Color bars represent T values. Error bars indicate SEM. au: arbitrary units, TBS: theta-burst stimulation, i: intermittent, c: continuous. 
in hippocampal and striatal networks during motor sequence learning. Our results showed that while the different stimulation conditions did not modulate motor behavior or brain activity in our ROIs, they altered the dynamical connectivity patterns in fronto-hippocampal and striatal networks during learning. Importantly, stimulation conditions differently influenced the relationship between changes in DLPFC GABA+ levels and both dynamical activity and connectivity patterns of the hippocampus and striatum during motor sequence learning. This research is, to our knowledge, the first to demonstrate that brain stimulation can influence motor learning-related responses in the striatum and the hippocampus.

\subsection{DLPFC stimulation influenced connectivity in fronto-hippocampal and striatal networks during motor sequence learning}

Brain imaging analyses revealed that DLPFC stimulation before learning altered connectivity in fronto-hippocampal and striatal networks during motor sequence learning. These results are in line with previous research in both the motor (Herz et al., 2014) and the declarative memory domain (Bilek et al., 2013; Esslinger et al., 2014; Davis et al., 2017) showing that frontal stimulation can alter task-related connectivity patterns of frontal areas with other cortical areas as well as with deep regions including the basal ganglia and the hippocampus.

Connectivity analyses using the stimulated DLPFC as a seed region indicated that after inhibitory cTBS and facilitatory iTBS, frontohippocampal connectivity increased and decreased, respectively, as a function of sequence learning. Interestingly, both activity and connectivity in hippocampo-frontal networks are usually described to decrease as a function of learning under normal (i.e., non-stimulated) conditions (Albouy et al., 2008, 2012, 2013a; Doyon et al., 2018). Our data therefore suggest that inhibitory cTBS disrupted the usually observed pattern of hippocampo-frontal responses during learning. Based on previous work proposing that the hippocampus, together with the fronto-parietal networks, supports early representations of motor sequences under high control and attentional processes (Hikosaka et al., 2002; Doyon et al., 2009, 2018; Albouy et al., 2013a), our connectivity results suggest that inhibitory cTBS might have altered these early control processes. Note that this interpretation remains hypothetical as the comparison of our results to a no stimulation condition is limited to the available literature as no sham condition was included in the current study. Interestingly, we showed in previous studies that hippocampal activity and connectivity patterns during initial motor sequence learning are critically linked to subsequent consolidation processes (Albouy et al., 2008, 2013a). It is therefore tempting to speculate that the stimulation-induced modulation of hippocampo-frontal responses might influence subsequent motor memory retention. While this remains hypothetical, it is indeed in line with earlier behavioral work showing that DLPFC stimulation can influence motor memory consolidation (Galea et al., 2010; Tunovic et al., 2014).

Striatal connectivity analyses indicated that facilitatory iTBS and inhibitory cTBS of the DLPFC promoted a progressive increase in connectivity within sensorimotor- and associative-striatal networks, respectively. Previous research has extensively described dynamical activity and connectivity patterns in striatal circuits during sequence learning (Hikosaka et al., 2002; Doyon et al., 2009; Albouy et al., 2013a). Task practice is usually paralleled by a gradual shift in activity from associative territories of the striatum which support slow and variable performance early during learning (Lehéricy et al., 2005; Albouy et al., 2012), to sensorimotor areas of the putamen when performance plateaus and automatization is reached (Lehéricy et al., 2005). Interestingly, the present results suggest that facilitatory iTBS to the DLPFC further promoted the practice-related shift to sensorimotor striatal functioning. In contrast, inhibitory cTBS altered the usually observed decrease in associative striatum involvement and induced learning-related increases in connectivity between the associative striatum (caudate nucleus) and the putamen. Together with the observation of inhibitory-stimulation- induced increases in fronto-hippocampal connectivity over the course of learning, the present results indicate that inhibitory prefrontal cTBS promoted the progressive engagement of networks involved in early learning and control processes. Future research is however warranted to confirm that these effects are purely induced by cTBS (as compared to sham stimulation) and not the result of the comparison of two active stimulation conditions. It is also worth noting that we did not observe any stimulation-induced changes in hippocampo-striatal functional connectivity as recently proposed by Freedberg and colleagues (Freedberg et al., 2020).

\subsection{DLPFC stimulation altered the relationship between DLPFC GABA+ levels and functional responses in the hippocampus and striatum}

GABA levels measured in cortical structures are assumed to reflect inhibitory GABAergic interneuron activity (Tremblay et al., 2016). Higher GABA levels would therefore result from higher GABAergic interneuron activity which in turn reflects lower activity of pyramidal neurons and therefore the local inhibition tone (Rae, 2014). In the present study, neither stimulation nor task conditions impacted DLPFC GABA+ levels. However, exploratory analyses within the facilitatory iTBS condition showed larger GABA+ decreases after sequential as compared to random task practice. We speculate that, similar to learning-induced decreases in M1 (Floyer-Lea et al., 2006; SampaioBaptista et al., 2015; Kolasinski et al., 2018), the decrease in DLPFC GABA+ levels might reflect disinhibition processes that promote successful learning (Stagg et al., 2011a; Kolasinski et al., 2018). This effect was observed under the effect of facilitatory stimulation, which might suggest that stimulation potentiated the neural plasticity processes. Although this interpretation is speculative given the absence of a stimulation by task interaction, it is in line with previous studies describing decreases of M1 GABA levels after facilitatory stimulation of M1 (Stagg et al., 2009a; Bachtiar et al., 2015, 2018).

Interestingly, our BOLD/GABA regression analyses showed that the type of stimulation applied before motor sequence learning affected the relationship between DLPFC GABA changes and (i) activity patterns in the hippocampus and the striatum and (ii) in DLPFC-striatumhippocampus connectivity (but see considerations below). These results provide direct support for a central role of the DLPFC in orchestrating the interaction between hippocampal and striatal systems during motor sequence learning (Albouy et al., 2013a). Importantly, the present data offer the first evidence that dynamical activity patterns of the hippocampus and striatum as well as fronto-hippocampo-striatal connectivity are related to the changes in inhibitory tone of the DLPFC. Our results also highlight the critical concept that the relationship between DLPFC GABA changes and functional responses in the hippocampus and the striatum can be altered by DLPFC stimulation. The absence of a sham condition however limits further interpretation regarding the effect of the different stimulation conditions on the relationship between DLPFC GABA and BOLD responses in deeper brain regions.

\subsection{DLPFC stimulation did not affect motor performance}

Prefrontal stimulation applied before motor sequence learning did not influence motor performance. Our findings raise the possibility that prefrontal stimulation might not be an effective avenue to modulate motor performance. This explanation is certainly possible, yet it is not in line with previous behavioral work showing that disruptive DLPFC stimulation applied before or during motor sequence learning can effectively impair motor performance and learning processes (Pascual-Leone et al., 1996; Robertson et al., 2001; Burke and Coats, 2016; Dayan et al., 2018). The discrepancy between these findings and our current results could be explained by several factors, including differences in stimulation procedure (e.g., TBS vs. $1 \mathrm{~Hz}, 5 \mathrm{~Hz}$ repetitive TMS or single pulse TMS), task complexity (bimanual vs. unimanual tasks), awareness of the sequential 
material to learn (explicit vs. implicit) and whether reward was provided or not during learning.

However, it is also possible that the absence of behavioral effects in the current study and therefore the disconnect between the effect of stimulation at the behavioral and brain levels could be the result of compensatory brain responses. One could have expected that, in the inhibitory stimulation condition, the prolonged engagement of associative striatal and fronto-hippocampal networks - usually observed early during learning when performance is poor - would result in slower performance. As no differences in motor behavior were observed between stimulation conditions, we propose that the sustained engagement of associative striatum-hippocampo-frontal areas during learning under inhibitory cTBS might represent a compensatory mechanism allowing performance to be maintained over the course of practice. The continued engagement of these regions may have counteracted the disruptive effect of stimulation on frontal control processes early during learning and thus may have contributed to improvements in performance during task practice despite a progressive decrease in connectivity within sensorimotor-striatal territories.

\subsection{Considerations}

Our results show that DLPFC stimulation did not influence activity patterns in our ROIs (but see Supplemental Table S13, Supplemental Fig. S4 and Supplemental Results for modulation in fronto-parietalcerebellar areas). This is partly in line with the available literature as there is, to our knowledge, no report of prefrontal-stimulation induced modulation of hippocampal activity [but see (Kim et al., 2018; Hermiller et al., 2020; Thakral et al., 2020) for parietal-stimulationinduced modulation of hippocampal activity] and only a few observations of activity changes in the striatum (Ott et al., 2011; van Holstein et al., 2018). This stands in contrast with extensive evidence of prefrontal-stimulation-induced modulation of functional connectivity, in particular between the cortical target and deeper brain regions (van der Werf et al., 2010; Bilek et al., 2013; Esslinger et al., 2014; Alkhasli et al., 2019; Shang et al., 2019; Tang et al., 2019). Together with these earlier observations, our results suggest that prefrontal stimulation can modulate connectivity patterns of the target and deeper brain regions without inducing changes in activity levels.

It is worth acknowledging that the present study did not include a sham stimulation condition. We made the methodological choice to compare two active stimulation conditions (i.e., iTBS and cTBS) rather than to include a sham stimulation condition as there is ongoing debate in the literature with respect to the appropriateness of sham stimulation for within-subject TMS protocols (Duecker and Sack, 2015; Bergmann and Hartwigsen, 2020). As a result, we decided to prioritize the inclusion of a control task condition rather than a control stimulation condition. The design therefore included a random task condition that afforded us with the opportunity to test for sequence learning-specific effects and to investigate whether the effect of stimulation on brain function depends on the "state" under which stimulation was active (i.e., learning vs. control). We are however aware of the limitations related to the lack of a sham control condition. Specifically, any significant results in the present study are derived from comparisons between two active stimulation conditions and not from contrasting each stimulation condition against baseline. A discussion of our results in the context of a no stimulation condition was therefore limited to qualitative comparisons with the available literature. Future research is therefore warranted to investigate whether the effects reported in this study are purely induced by stimulation (as compared to sham) and not the result of the comparison of two active stimulation conditions.

While MRS-derived GABA levels have been associated to plasticity processes (Floyer-Lea et al., 2006; Stagg et al., 2009b, 2009a, 2011a; Marjańska et al., 2013; Bachtiar et al., 2015, 2018; SampaioBaptista et al., 2015; Kolasinski et al., 2018), there is some ongoing debate on what these measures reflect. Previous studies suggest that GABA levels quantified with MRS reflect extracellular GABA (Stagg et al., 2011b; Dyke et al., 2017) but it is generally accepted that GABA+ levels cannot be clearly assigned to one of the various pools of GABA found in the brain [see (Stagg et al., 2011a,b; Stagg, 2014)]. Furthermore, due to issues with data quality, we were not able to investigate the effects of our intervention on GABA+ levels in the hippocampus. Additionally, we did not include measurements of striatal GABA due to time constraints imposed by the experimental design. Given the critical roles of these structures in motor sequence learning, it would be of interest for future research to examine learning- and stimulation-induced effects on striatal and hippocampal GABA. Last, and perhaps most importantly, given our within-subject design and the corresponding statistical models necessary to investigate the relationship between BOLD and GABA+ data, it is not possible to provide a directional interpretation of the regression results. Specifically, a significant effect in such an analysis represents a between stimulation condition difference in the relationships between: a) learning-dependent modulations in brain activity/connectivity (referred to as differential modulation betas; depicted on the y-axes on Fig. 5); and, b) $\triangle$ GABA across the stimulation/task interval (i.e., differential $\triangle \mathrm{GABA}$; $\mathrm{x}$-axes on Fig. 5). As the beta estimates representing the modulation in brain activity/connectivity as well as $\triangle \mathrm{GABA}$ are both bi-directional (i.e., values represent an increase or decrease in activity/connectivity with learning or an increase or decrease in GABA+ after the intervention), the difference between stimulation conditions computed on these parameters could then reflect various individual patterns. For example, a large differential modulation beta could be attributed to a steeper decrease in activity in iSEQ than cSEQ or to no modulation in iSEQ and an increase in activity in cSEQ. A deeper inspection of these various possibilities revealed no single pattern that could adequately summarize the reported effects.

\section{Conclusions}

In the present proof-of-concept study that employed a multimodal neuroimaging approach, we demonstrated that DLPFC stimulation influenced connectivity patterns within hippocampo-frontal and striatal networks during motor sequence learning. Our data also showed that non-invasive brain stimulation altered the relationship between the levels of inhibition, as assessed with MRS of GABA, in the stimulated area and learning-related changes in both activity and connectivity in frontostriato-hippocampal networks. This provides the first experimental evidence, to the best of our knowledge, that prefrontal brain stimulation can alter functional responses in the striatum and hippocampus during motor learning.

\section{Author contributions}

Mareike Gann: Conceptualization; Resources; Data curation; Software; Formal analysis; Investigation; Visualization; Writing - original draft; Writing - review and editing Bradley King: Conceptualization; Resources; Data curation; Software; Formal analysis; Validation; Writing - review and editing Nina Dolfen: Investigation; Methodology; Writing - review and editing Menno Veldman: Investigation; Writing - review and editing Kimberly Chan: Resources; Software; Methodology; Writing - review and editing Nicolaas Puts: Resources; Software; Methodology; Writing - review and editing Richard Edden: Resources; Software; Methodology; Writing - review and editing Marco Davare: Conceptualization; Methodology; Writing - review and editing Stephan Swinnen: Methodology; Writing - review and editing Dante Mantini: Resources; Software; Methodology; Writing - review and editing Edwin Robertson: Conceptualization; Writing - review and editing Genevieve Albouy: Conceptualization; Resources; Data curation; Software; Formal analysis; Supervision; Funding acquisition; Validation; Investigation; Writing - original draft; Project administration; Writing - review and editing 


\section{Data Availability}

The ethical approval granted by the local ethics committee does not permit the publication of data online.

\section{Declaration of Competing Interest}

The authors have no conflict of interest to declare.

\section{Acknowledgements}

This work was supported by the Belgian Research Foundation Flanders (FWO; G099516N) and internal funds from KU Leuven. GA also received support from FWO (G0D7918N, G0B1419N, 1524218N) and Excellence of Science (EOS, 30446199, MEMODYN, with SPS and DM). MAG, ND and MPV received salary support from these grants. MAG is funded by a predoctoral fellowship from FWO (1141320N). Financial support for author BRK was provided by the European Union's Horizon 2020 research and innovation program under the Marie SkłodowskaCurie grant agreement (703490) and a postdoctoral fellowship from FWO (132635). This study applies tools developed under National Institutes of Health (NIH) Grants R01- EB-016089, R01-023963, and P41EB015909; RAEE also receives salary support from these grants. NAJP receives salary support from NIH Grant R00-MH-107719. EMR received salary support from the Air Force Office of Scientific Research (AFOSR, Virginia, USA; FA9550-16-1-0191). We wish to thank Kaat van Rooij and Michelle Roussard for assistance with data collection.

\section{Supplementary materials}

Supplementary material associated with this article can be found, in the online version, at doi:10.1016/j.neuroimage.2021.118158.

\section{References}

Albouy, G, King, BR, Maquet, P, Doyon, J, 2013a. Hippocampus and striatum: dynamics and interaction during acquisition and sleep-related motor sequence memory consolidation. Hippocampus 23, 985-1004.

Albouy, G, Sterpenich, V, Balteau, E, Vandewalle, G, Desseilles, M, Dang-Vu, T, Darsaud, A, Ruby, P, Luppi, PH, Degueldre, C, Peigneux, P, Luxen, A, Maquet, P, 2008. Both the hippocampus and striatum are involved in consolidation of motor sequence memory. Neuron 58, 261-272.

Albouy, G, Sterpenich, V, Vandewalle, G, Darsaud, A, Gais, S, Rauchs, G, Desseilles, M, Boly, M, Dang-Vu, T, Balteau, E, Degueldre, C, Phillips, C, Luxen, A, Maquet, P, 2012. Neural correlates of performance variability during motor sequence acquisition. Neuroimage 60, 324-331. Available at: doi: 10.1016/j.neuroimage.2011.12.049.

Albouy, G, Sterpenich, V, Vandewalle, G, Darsaud, A, Gais, S, Rauchs, G, Desseilles, M, Boly, M, Dang-Vu, T, Balteau, E, Degueldre, C, Phillips, C, Luxen, A, Maquet, P, 2013b. Interaction between Hippocampal and striatal systems predicts subsequent consolidation of motor sequence memory. PLoS One 8, 12-14.

Alkhasli, I, Sakreida, K, Mottaghy, FM, Binkofski, F, 2019. Modulation of Fronto-Striatal functional connectivity using transcranial magnetic stimulation. Front. Hum. Neurosci. 13, 190. Available at: https://www.frontiersin.org/article/10.3389/fnhum.2019.00190/full .

Bachtiar, V, Johnstone, A, Berrington, A, Lemke, C, Johansen-Berg, H, Emir, U, Stagg, CJ, 2018. Modulating regional motor cortical excitability with noninvasive brain stimulation results in neurochemical changes in bilateral motor cortices. J. Neurosci. 38, 7327-7336.

Bachtiar, V, Near, J, Johansen-Berg, H, Stagg, CJ, 2015. Modulation of GABA and resting state functional connectivity by transcranial direct current stimulation. eLife 4, 1-9.

Beck, AT, Epstein, N, Brown, G, Steer, RA, 1988. An inventory for measuring clinical anxiety: psychometric properties. J. Consult. Clin. Psychol. 56, 893-897.

Beck, AT, Ward, CH, Mendelson, M, Mock, J, Erbaugh, J, 1961. An inventory for measuring depression. Arch. Gen. Psychiatry 4, 561-571. Available at: doi:10.1001/archpsyc.1961.01710120031004.

Bergmann, TO, Hartwigsen, G, 2020. Inferring causality from noninvasive brain stimulation in cognitive neuroscience. J. Cognit. Neurosci. 1-29.

Beynel, L, Appelbaum, LG, Luber, B, Crowell, CA, Hilbig, SA, Lim, W, Nguyen, D, Chrapliwy, NA, Davis, SW, Cabeza, R, Lisanby, SH, De, Deng Z, 2019. Effects of online repetitive transcranial magnetic stimulation (rTMS) on cognitive processing: a meta-analysis and recommendations for future studies. Neurosci. Biobehav. Rev. 107, 47-58.

Bilek, E, Schafer, A, Ochs, E, Esslinger, C, Zangl, M, Plichta, MM, Braun, U, Kirsch, P, Schulze, TG, Rietschel, M, Meyer-Lindenberg, A, Tost, H, 2013. Application of highfrequency repetitive transcranial magnetic stimulation to the DLPFC alters human prefrontal-hippocampal functional interaction. J. Neurosci. 33, 7050-7056. Available at: http://www.jneurosci.org/content/33/16/7050.full.pdf
Brainard, DH, 1997. The psychophysics toolbox. Spat. Vis. 10, 433-436.

Burke, MR, Coats, RO, 2016. Dissociation of the rostral and dorsolateral prefrontal cortex during sequence learning in saccades: a TMS investigation. Exp. Brain Res. 234, 597-604.

Buysse, DJ, Reynolds, CF, Monk, TH, Berman, SR, Kupfer, DJ, 1989. The Pittsburgh sleep quality index: a new instrument for psychiatric practice and research. Psychiatry Res. 28, 193-213.

Chung, SW, Lewis, BP, Rogasch, NC, Saeki, T, Thomson, RH, Hoy, KE, Bailey, NW, Fitzgerald, PB, 2017. Demonstration of short-term plasticity in the dorsolateral prefrontal cortex with theta burst stimulation: a TMS-EEG study. Clin. Neurophysiol. 128, 1117-1126.

Davis, SW, Luber, B, Murphy, DLK, Lisanby, SH, Cabeza, R, 2017. Frequency-specific neuromodulation of local and distant connectivity in aging and episodic memory function. Hum. Brain Mapp. 38, 5987-6004.

Dayan, E, Herszage, J, Laor-Maayany, R, Sharon, H, Censor, N, 2018. Neuromodulation of reinforced skill learning reveals the causal function of prefrontal cortex. Hum. Brain Mapp. 39, 4724-4732. Available at: http://doi.wiley.com/10.1002/hbm.24317.

de Beukelaar, TT, Van Soom, J, Huber, R, Wenderoth, N, 2016. A day awake attenuates motor learning-induced increases in corticomotor excitability. Front. Hum. Neurosci. $10,138$.

Doyon, J, Bellec, P, Amsel, R, Penhune, V, Monchi, O, Carrier, J, Lehéricy, S, Benali, H, 2009. Contributions of the basal ganglia and functionally related brain structures to motor learning. Behav. Brain Res. 199, 61-75.

Doyon, J, Gabitov, E, Vahdat, S, Lungu, O, Boutin, A, 2018. Current issues related to motor sequence learning in humans. Curr. Opin. Behav. Sci. 20, 89-97. Available at: http://linkinghub.elsevier.com/retrieve/pii/S2352154617301213.

Duecker, F, Sack, AT, 2015. Rethinking the role of sham TMS. Front. Psychol. 6, 210.

Duncan, NW, Wiebking, C, Northoff, G, 2014. Associations of regional GABA and glutamate with intrinsic and extrinsic neural activity in humans-A review of multimodal imaging studies. Neurosci. Biobehav. Rev. 47, 36-52.

Dyke, K., Pépés, S.E., Chen, C., Kim, S., Sigurdsson, H.P., Draper, A., Husain, M., Nachev, P., Gowland, P.A., Morris, P.G., Jackson, S.R., 2017. Comparing GABAdependent physiological measures of inhibition with proton magnetic resonance spectroscopy measurement of GABA using ultra-high-field MRI. NeuroImage 152, 360370. Available at: doi: 10.1016/j.neuroimage.2017.03.011.

Edden, RAE, Oeltzschner, G, Harris, AD, Puts, NAJ, Chan, KL, Boer, VO, Schär, M, Barker, PB, 2016. Prospective frequency correction for macromolecule suppressed GABA editing experiments at 3T. J. Magn. Reson. Imaging 44, 1474-1482.

Edden, RAE, Puts, NAJ, Barker, PB, 2012. Macromolecule-suppressed GABA-edited magnetic resonance spectroscopy at 3T. Magn. Reson. Med. 68, 657-661.

Edden, RAE, Puts, NAJ, Harris, AD, Barker, PB, Evans, CJ, 2014. Gannet: a batch-processing tool for the quantitative analysis of gamma-aminobutyric acid-edited MR spectroscopy spectra. J. Magn. Reson. Imaging 40, 1445-1452.

Ellis, B, Johns, M, Lancaster, R, Raptopoulos, P, Angelopoulos, N, Priest, R, 1981. The St. Mary's hospital sleep questionnaire: a study of reliability. Sleep 4, 93-97.

Esslinger, C, Schüler, N, Sauer, C, Gass, D, Mier, D, Braun, U, Ochs, E, Schulze, TG, Rietschel, M, Kirsch, P, Meyer-Lindenberg, A, 2014. Induction and quantification of prefrontal cortical network plasticity using $5 \mathrm{~Hz}$ rTMS and fMRI. Hum. Brain Mapp. $35,140-151$.

Floyer-Lea, A, Wylezinska, M, Kincses, T, Matthews, PM, 2006. Rapid modulation of GABA concentration in human sensorimotor cortex during motor learning. J. Neurophysiol. $95,1639-1644$.

Fox, MD, Buckner, RL, White, MP, Greicius, MD, Pascual-Leone, A, 2012a. Efficacy of transcranial magnetic stimulation targets for depression is related to intrinsic functional connectivity with the subgenual cingulate. Biol. Psychiatry 72, 595-603.

Fox, MD, Halko, MA, Eldaief, MC, Pascual-Leone, A, 2012b. Measuring and manipulating brain connectivity with resting state functional connectivity magnetic resonance imaging (fcMRI) and transcranial magnetic stimulation (TMS). Neuroimage 62, 2232-2243.

Freedberg, M, Reeves, JA, Toader, AC, Hermiller, MS, Voss, JL, Wassermann, EM, 2019. Persistent enhancement of hippocampal network connectivity by parietal rTMS is reproducible. eNeuro $6,1-13$

Freedberg, M, Toader, AC, Wassermann, EM, Voss, JL, 2020. Competitive and cooperative interactions between medial temporal and striatal learning systems. Neuropsychologia 136, 107257.

Galea, JM, Albert, NB, Ditye, T, Miall, RC, 2010. Disruption of the dorsolateral prefrontal cortex facilitates the consolidation of procedural skills. J. Cognit. Neurosci. $22,1158-1164$.

Gitelman, DR, Penny, WD, Ashburner, J, Friston, KJ, 2003. Modeling regional and psychophysiologic interactions in fMRI: The importance of hemodynamic deconvolution. Neuroimage 19, 200-207.

Goldsworthy, MR, Hordacre, B, Ridding, MC, 2016. Minimum number of trials required for within- and between-session reliability of TMS measures of corticospinal excitability. Neuroscience 320, 205-209. Available at: doi:10.1016/j.neuroscience.2016.02.012.

Hanlon, CA, Dowdle, LT, Moss, H, Canterberry, M, George, MS, 2016. Mobilization of medial and lateral frontal-striatal circuits in cocaine users and controls: an interleaved TMS/BOLD functional connectivity study. Neuropsychopharmacology 41 , 3032-3041.

Harris, AD, Glaubitz, B, Near, J, John Evans, C, Puts, NAJ, Schmidt-wilcke, T, Tegenthoff, M, Barker, PB, Edden, RAE, 2014. The impact of frequency drift on GABA-edited MR spectroscopy. Magn. Reson. Med. 72, 941-948.

Harris, AD, Puts, NAJ, Edden, RAE, 2015. Tissue correction for GABA-edited MRS: considerations of voxel composition, tissue segmentation, and tissue relaxations. J. Magn. Reson. Imaging 42, 1431-1440.

Hermans, L, Levin, O, Maes, C, van Ruitenbeek, P, Heise, KF, Edden, RAE, Puts, NAJ, Peeters, R, King, BR, Meesen, RLJ, Leunissen, I, Swinnen, SP, Cuypers, K, 2018. GABA 
levels and measures of intracortical and interhemispheric excitability in healthy young and older adults: an MRS-TMS study. Neurobiol. Aging 65, 168-177.

Hermiller, MS, Chen, YF, Parrish, TB, Voss, JL, 2020. Evidence for immediate enhancement of hippocampal memory encoding by network-targeted theta-burst stimulation during concurrent fMRI. J. Neurosci. 40 JN-RM-0486-20.

Herz, DM, Christensen, MS, Bruggemann, N, Hulme, OJ, Richard Ridderinkhof, K, Madsen, KH, Siebner, HR, 2014. Motivational tuning of fronto-subthalamic connectivity facilitates control of action impulses. J. Neurosci. 34, 3210-3217.

Hikosaka, O, Nakamura, K, Sakai, K, Nakahara, H, 2002. Central mechanisms of motor skill learning. Proc. SID 31, 267-365.

Holm, S, 1979. A simple sequentially rejective multiple test procedure. Scand. J. Stat. 6, 65-70.

Hone-Blanchet, A, Edden, RA, Fecteau, S, 2016. Online effects of transcranial direct current stimulation in real time on human prefrontal and striatal metabolites. Biol. Psychiatry 80, 432-438.

Huang, YZ, Edwards, MJ, Rounis, E, Bhatia, KP, Rothwell, JC, 2005. Theta burst stimulation of the human motor cortex. Neuron 45, 201-206.

Huang, YZ, Rothwell, JC, Edwards, MJ, Chen, RS, 2008. Effect of physiological activity on an NMDA-dependent form of cortical plasticity in human. Cereb. Cortex 18, 563-570.

Iwabuchi, SJ, Raschke, F, Auer, DP, Liddle, PF, Lankappa, ST, Palaniyappan, L, 2017. Targeted transcranial theta-burst stimulation alters fronto-insular network and prefrontal GABA. Neuroimage 146, 395-403.

Kim, S, Nilakantan, AS, Hermiller, MS, Palumbo, RT, VanHaerents, S, Voss, JL, 2018. Selective and coherent activity increases due to stimulation indicate functional distinctions between episodic memory networks. Sci. Adv. 4, 1-10.

King, BR, Dolfen, N, Gann, MA, Renard, Z, Swinnen, SP, Albouy, G, 2019. Schema and motor-memory consolidation. Psychol. Sci. 30, 963-978.

King, BR, Rumpf, JJ, Verbaanderd, E, Heise, KF, Dolfen, N, Sunaert, S, Doyon, J, Classen, J, Mantini, D, Puts, NAJ, Edden, RAE, Albouy, G, Swinnen, SP, 2020. Baseline sensorimotor GABA levels shape neuroplastic processes induced by motor learning in older adults. Hum. Brain Mapp. 41, 3680-3695.

King, BR, van Ruitenbeek, P, Leunissen, I, Cuypers, K, Heise, KF, Santos Monteiro, T, Hermans, L, Levin, O, Albouy, G, Mantini, D, Swinnen, SP, 2018. Age-related declines in motor performance are associated with decreased segregation of large-scale resting state brain networks. Cereb. Cortex.

Kolasinski, J, Hinson, EL, Divanbeighi Zand, AP, Rizov, A, Emir, UE, Stagg, CJ, 2018. The dynamics of cortical GABA in human motor learning. J. Physiol. 1, 271-282.

Lehéricy, S, Benali, H, Van de Moortele, P-F, Pélégrini-Issac, M, Waechter, T, Ugurbil, K, Doyon, J, 2005. Distinct basal ganglia territories are engaged in early and advanced motor sequence learning. PNAS 102, 12566-12571. Available at: http://www.ncbi.nlm.nih.gov/pubmed/16107540\%0Ahttp://www.pubmedcentral. nih.gov/articlerender.fcgi?artid=PMC1194910

Lehéricy, S, Ducros, M, Van De Moortele, PF, Francois, C, Thivard, L, Poupon, C, Swindale, N, Ugurbil, K, Kim, DS, 2004. Diffusion tensor fiber tracking shows distinct corticostriatal circuits in humans. Ann. Neurol. 55, 522-529.

Maclean, AW, Fekken, GC, Saskin, P, Knowles, JB, 1992. Psychometric evaluation of the Stanford Sleepiness Scale. J. Sleep Res. 1, 35-39.

Maes, C, Hermans, L, Pauwels, L, Chalavi, S, Leunissen, I, Levin, O, Cuypers, K, Peeters, R, Sunaert, S, Mantini, D, Puts, NAJ, Edden, RAE, Swinnen, SP, 2018. Age-related differences in GABA levels are driven by bulk tissue changes. Hum. Brain Mapp. 39, 3652-3662.

Marjańska, M, Lehéricy, S, Valabregue, R, Popa, T, Bonnet, C, Gallea, C, Coudert, M, 2013. Brain dynamic neurochemical changes in dystonic patients: a magnetic resonance spectroscopy study. Mov. Disord. 28, 201-209.

Mescher, M, Merkle, H, Kirsch, J, Garwood, M, Gruetter, R, 1998. Simultaneous in vivo spectral editing and water suppression. NMR Biomed. 11, 266-272.

Mikkelsen, M, et al., 2019. Big GABA II: water-referenced edited MR spectroscopy at 25 research sites. Neuroimage 191, 537-548.

Mullins, PG, McGonigle, DJ, O'Gorman, RL, Puts, NAJ, Vidyasagar, R, Evans, CJ, ..., Edden, RAE, 2014. Current practice in the use of MEGA-PRESS spectroscopy for the detection of GABA. Neuroimage 86, 43-52.

Muto, V, Jaspar, M, Meyer, C, Kussé, C, Chellappa, SL, Degueldre, C, Balteau, E, Shaffii-Le Bourdiec, A, Luxen, A, Middleton, B, Archer, SN, Phillips, C, Collette, F, Vandewalle, G, Dijk, D-J, Maquet, P, 2016. Local modulation of human brain responses by circadian rhythmicity and sleep debt. Science 353, 687 LP-690.

Near, J, Edden, R, Evans, CJ, Paquin, R, Harris, A, Jezzard, P, 2015. Frequency and phase drift correction of magnetic resonance spectroscopy data by spectral registration in the time domain. Magn. Reson. Med. 73, 44-50.

Nichols, N. http://www2.warwick.ac.uk/fac/sci/statistics/staff/academic-research/ nichols/scripts/fsl/.

Nissen, MJ, Bullemer, P, 1987. Attentional requirements of learning: Evidence from performance measures. Cognit. Psychol. 19, 1-32.

Oldfield, RC, 1971. The assessment and analysis of handedness: the Edinburgh inventory. Neuropsychologia 9, 97-113

Ott, DVM, Ullsperger, M, Jocham, G, Neumann, J, Klein, TA, 2011. Continuous theta-burst stimulation (cTBS) over the lateral prefrontal cortex alters reinforcement learning bias. Neuroimage 57, 617-623. Available at: doi:10.1016/j.neuroimage.2011.04.038.
Pascual-Leone, A, Wassermann, EM, Grafman, J, Hallett, M, 1996. The role of the dorsolateral prefrontal cortex in implicit procedural learning. Exp. Brain Res. 107, 479-485.

Penhune, VB, Steele, CJ, 2012. Parallel contributions of cerebellar, striatal and M1 mechanisms to motor sequence learning. Behav. Brain Res. 226, 579-591. Available at: doi:10.1016/j.bbr.2011.09.044.

Poldrack, RA, 2007. Region of interest analysis for fMRI. Soc. Cognit. Affect. Neurosci. 2, 67-70.

Poldrack, RA, Fletcher, PC, Henson, RN, Worsley, KJ, Brett, M, Nichols, TE, 2008. Guidelines for reporting an fMRI study. Neuroimage 40, 409-414.

Puts, NAJ, Edden, RAE, 2012. In vivo magnetic resonance spectroscopy of GABA: a methodological review. Prog. Nucl. Magn. Reson. Spectrosc. 60, 29-41.

Robertson, EM, Tormos, JM, Maeda, F, Pascual-Leone, A, 2001. The role of the dorsolateral prefrontal cortex during sequence learning is specific for spatial information. Cereb. Cortex 11, 628-635.

Rothman, DL, Petroff, OAC, Behar, KL, Mattson, RH, 1993. Localized 1H NMR measurements of $\gamma$-aminobutyric acid in human brain in vivo. PNAS 90, 5662-5666.

Sack, AT, Kadosh, RC, Schuhmann, T, Moerel, M, Walsh, V, Goebel, R, 2009. Optimizing functional accuracy of TMS in cognitive studies: a comparison of methods. J. Cognit. Neurosci. 21, 207-221.

Sampaio-Baptista, C, Filippini, N, Stagg, CJ, Near, J, Scholz, J, Johansen-Berg, H, 2015. Changes in functional connectivity and GABA levels with long-term motor learning. Neuroimage 106, 15-20. Available at: doi:10.1016/j.neuroimage.2014.11.032.

Shang, Y, Chang, D, Zhang, J, Peng, W, Song, D, Gao, X, Wang, Z, 2019. Theta-burst transcranial magnetic stimulation induced functional connectivity changes between dorsolateral prefrontal cortex and default-mode-network. Brain Imaging Behav..

Smarr, BL, Jennings, KJ, Driscoll, JR, Kriegsfeld, LJ, 2014. A time to remember: the role of circadian clocks in learning and memory. Curr. Opin. Biotechnol. 29, 146-155.

Stagg, CJ, 2014. Magnetic Resonance Spectroscopy as a tool to study the role of GABA in motor-cortical plasticity. Neuroimage 86, 19-27. Available at: doi:10.1016/j.neuroimage.2013.01.009.

Stagg, CJ, Bachtiar, V, Johansen-Berg, H, 2011a. The role of GABA in human motor learning. Curr. Biol. 21, 480-484. Available at: doi:10.1016/j.cub.2011.01.069.

Stagg, CJ, Best, JG, Stephenson, MC, O'Shea, J, Wylezinska, M, Kineses, ZT, Morris, PG, Matthews, PM, Johansen-Berg, H, 2009a. Polarity-sensitive modulation of cortical neurotransmitters by transcranial stimulation. J. Neurosci. 29, 5202-5206.

Stagg, CJ, Bestmann, S, Constantinescu, AO, Moreno Moreno, L, Allman, C, Mekle, R, Woolrich, M, Near, J, Johansen-Berg, H, Rothwell, JC, 2011b. Relationship between physiological measures of excitability and levels of glutamate and GABA in the human motor cortex. J. Physiol. 589.

Stagg, CJ, Wylezinska, M, Matthews, PM, Jezzard, P, Rothwell, JC, Bestmann, S, 2009b. Neurochemical effects of theta burst stimulation as assessed by magnetic resonance spectroscopy. J. Neurophysiol. 101, 2872-2877.

Steele, CJ, Penhune, VB, 2010. Specific increases within global decreases: a functional magnetic resonance imaging investigation of five days of motor sequence learning. J. Neurosci. 30, 8332-8341.

Tambini, A, Nee, DE, D'Esposito, M, 2018. Hippocampal-targeted theta-burst stimulation enhances associative memory formation. J. Cognit. Neurosci. 30, 1452-1472.

Tang, Y, Jiao, X, Wang, J, Zhu, T, Zhou, J, Qian, Z, Zhang, T, Cui, H, Li, H, Tang, X, Xu, L, Zhang, L, Wei, Y, Sheng, J, Liu, L, Wang, J, 2019. Dynamic functional connectivity within the Fronto-Limbic network induced by intermittent theta-burst stimulation: a pilot study. Front. Neurosci. 13, 944.

Thakral, PP, Madore, KP, Kalinowski, SE, Schacter, DL, 2020. Modulation of hippocampal brain networks produces changes in episodic simulation and divergent thinking. PNAS 117, 12729-12740.

Tremblay, R, Lee, S, Rudy, B, 2016. Properties to circuits. Neuron 91, 260-292.

Tunovic, S, Press, DZ, Robertson, EM, 2014. A physiological signal that prevents motor skill improvements during consolidation. J. Neurosci. 34, 5302-5310. Available at: http://www.jneurosci.org/cgi/doi/10.1523/JNEUROSCI.3497-13.2014

Tzourio-Mazoyer, N, Landeau, B, Papathanassiou, D, Crivello, F, Etard, O, Delcroix, N, Mazoyer, B, Joliot, M, 2002. Automated anatomical labeling of activations in SPM using a macroscopic anatomical parcellation of the MNI MRI single-subject brain. Neuroimage 15, 273-289.

van der Werf, YD, Sanz-Arigita, EJ, Menning, S, van den Heuvel, O.a, 2010. Modulating spontaneous brain activity using repetitive transcranial magnetic stimulation. BMC Neurosci. 11, 145. Available at: http://www.biomedcentral.com/1471-2202/11/145

van Holstein, M, Froböse, MI, O'Shea, J, Aarts, E, Cools, R, 2018. Controlling striatal function via anterior frontal cortex stimulation. Sci. Rep. 8, 1-13.

van Polanen, V, Rens, G, Davare, M, 2020. The role of the anterior intraparietal sulcus and the lateral occipital cortex in fingertip force scaling and weight perception during object lifting. J. Neurophysiol. Available at: doi:10.1152/jn.00771.2019.

Wang, JX, Rogers, LM, Gross, EZ, Ryals, AJ, Dokucu, ME, Brandstatt, KL, Hermiller, MS, Voss, JL, 2014. Targeted enhancement of cortical-hippocampal brain networks and associative memory. Science 345, 1054-1057 Available at: http://science.sciencemag.org/content/345/6200/1054.abstract. 